\title{
Optimal power policies and throughput scaling analyses in fading cognitive broadcast channels with primary outage probability constraint
}

\author{
Athipat Limmanee ${ }^{1}$, Subhrakanti Dey ${ }^{2 *}$ and Ehsan Nekouei ${ }^{3}$
}

\begin{abstract}
This paper focuses on a spectrum-sharing-based fading cognitive radio broadcast channel (BC) with a single-antenna secondary base station (SBS) and $M$ single-antenna secondary receivers (SRs) utilizing the same spectrum band with a delay-sensitive primary user (PU). The service-quality requirement for the primary user is set by an outage probability constraint (POC). We address the optimal power allocation problem for the SBS ergodic sum capacity (ESC) maximization in the secondary BC network subject to POC and a transmit power constraint at SBS specified by either a long-term or a short-term power constraint. The optimality conditions reveal that in each joint channel state, the SBS allocates transmission power to the only one selected SR with the highest value of a certain metric consisting of the ratio of the SR's direct channel power gain and the sum of interference power and noise power at the SR. Then, the secondary network throughput scaling analysis as the number of SRs becomes large, is also investigated, showing that if PU applies a truncated channel inversion (TCI) power policy, the SBS ESC scales like $\epsilon_{p} \log (\log M)$ where $\epsilon_{p}$ is the PU outage probability threshold. To reduce the amount of channel side information (CSI) transferred between the two networks, we propose a suboptimal transmission scheme which requires only 1-bit feedback from the delay-sensitive PR (partial CSI). We show that the new power control policy is asymptotically optimal, i.e. the SBS ESC under this reduced feedback scheme still scales like $\epsilon_{p} \log (\log M)$.
\end{abstract}

Keywords: Cognitive radio; Power control; Ergodic capacity; Outage probability; Throughput scaling; Fading broadcast channels

\section{Introduction}

Inefficiency of spectrum usage has become one of the main concerns in wireless communications technology today as most of the exclusively allocated spectrum remains underutilized by licensed/primary users (PUs) [1]. This has inspired the concept of cognitive radio (CR) technology, which was originally proposed in [2]. The rationale is that unlicensed/secondary users (SUs) are allowed to use the same spectrum with PUs as long as the quality-of-service (QoS) of the primary transmission is protected. To date, there are three main approaches for CR to manage resultant interference: interweave, overlay,

\footnotetext{
*Correspondence: subhra.dey@signal.uu.se

2 Department of Engineering Science, Uppsala University, P.O. Box 534 Uppsala 751 21, Sweden
}

Full list of author information is available at the end of the article and underlay [3]. In the underlay paradigm which is the paradigm of interest in this paper, SUs can share the spectrum regardless of the ON/OFF status of the primary network, providing that the QoS of primary link is still guaranteed. To protect the service quality of the primary transmission in underlay paradigm, several types of constraints have been proposed in literature including peak/average interference power constraint (PIPC/AIPC), primary capacity loss constraint, and primary outage probability constraint (see [4] and references therein).

Optimal power allocation strategy for non-cognitive BC has been extensively investigated under various perspectives, such as achievable ergodic capacity [5] and outage capacity [6] regions in fading broadcast channels or the optimal power allocation for maximizing ergodic sum 
downlink capacity in non-cognitive $\mathrm{BC}$ [7]. However, in a multi-user cognitive environment, optimal power allocation problems are even more challenging because of the strict guarantee of the QoS constraint in primary networks, especially in delay-sensitive applications. Furthermore, the asymptotic behaviour of the secondary sum downlink throughput scaling under the optimal power allocation policy, termed as optimal throughput scaling, when the number of secondary receivers grows large, has not been previously addressed under a primary outage probability constraint. Thus motivated, our first aim is to provide an analysis of optimal throughput scaling with full channel side information (CSI) at the secondary transmitter. While the requirement of full CSI at the SU transmitter, in particular, those of the primary channels is impractical. Nonetheless, these results will constitute a benchmark for assessing the performance of any other suboptimal power allocation scheme which may be designed in practical scenarios with partial channel side information about primary and secondary channels. Indeed, our second aim in this paper is to analyze secondary throughput scaling with a suboptimal power allocation scheme that only requires partial information about the channels involving only the primary terminals (see Section 6 for further details).

\subsection{Contributions}

In this paper, we focus on a single-input single-output (SISO) fading cognitive broadcast channel (C-BC) with $M$ SUs, co-existing with a delay-sensitive primary link under average and peak transmit power constraint at the secondary base station (SBS). The novel contributions of this paper can be summarized as follows:

1. We derive the optimal power control policy for the ergodic sum capacity (ESC) maximization problem under a PU outage probability constraint (POC), under both long-term and short-term power constraints at the SBS transmitter assuming perfect knowledge of all involved channel gains at the SBS along with the PU's transmission power control policy. (A typical PU is oblivious to the secondary network and thus its power control policy only depends on the direct gain between primary terminals rather than interferences from the secondary network.)

(a) Since the primary outage probability constraint has no closed-form expression in general, the MAC-BC duality result from [8] cannot be applied directly. Different from $[8,9]$, we propose a novel technique to establish the optimality of an opportunistic scheduling structure that maximizes the SBS ergodic sum rate. This result is then used to transform the originally non-convex ESC maximization problem to a convex optimization problem.

(b) We solve the transformed convex optimization problem by a probabilistic power allocation technique [10,11]. Our optimal power control solutions can be adapted to any arbitrary power control employed by the primary network.

2. We derive asymptotic scaling laws (also known as multi-user diversity gains) for the SBS ergodic sum capacity as the number of SUs, $M$, becomes large when SBS employs the optimal power allocation policy, assuming all channels undergo independent Rayleigh fading. In this part, we additionally presume that the delay-sensitive PU uses a truncated channel inversion (TCI) policy. The significance of choosing TCI policy is that it minimizes the primary outage probability in the absence of the secondary network (see [12]). Under either long-term or short-term power constraint at the SBS, we show that the SBS ESC scales according to $\epsilon_{p} \log (\log M)$ with a pre-log factor $\epsilon_{p}$, where $\epsilon_{p}$ is the maximum allowable outage probability at the primary receiver in the presence of the SU network.

It is worth mentioning that the current paper is different from [13] in three aspects. First, we assume that the delay-sensitive primary user employs an optimal power control policy for minimizing outage probability, i.e. truncated channel inversion power control policy. Second, we derive the optimal power control policy at the SBS under two different scenarios, i.e. SBS employs (i) a long-term power control policy or (ii) a short-term power control policy. Third, for each scenario, we study throughput scaling behaviour of the secondary network under the optimal power allocation policy at the SBS. Also, our throughput scaling results differ from [13] in a pre-log factor. This pre-log factor captures the effect of the optimal power control policy at the primary side on the throughput scaling behaviour of the secondary network.

3. We also propose a suboptimal transmission scheme, namely, a 1-bit primary feedback scheme, which substantially decreases the cooperation between the primary and the secondary networks compared to the optimal power allocation scheme. More specifically, we show that the secondary network just needs 1-bit feedback from the primary receiver to guarantee the QoS in the primary, while the multi-user diversity gain of the suboptimal scheme remains the same as the optimal one. 


\subsection{Outline of the paper}

The remainder of this paper is organized as follows. Related previous works are discussed in Section 2. The description of our system model is presented in Section 3. Under the assumption of full channel side information at SBS and PU's power strategy being known to SBS, the optimal power control policies for ESC maximizing problem with POC and either long-term transmit power constraint (LTPC) or short-term transmit power constraint (STPC) are presented in Section 4. In Section 5, we derive the optimal throughput scaling law of $\epsilon_{p} \log (\log M)$ under additional assumptions that all channels involved in the problem undergo independent and identically distributed (iid) Rayleigh fading and the PU employs a TCI power control policy. A suboptimal transmission scheme for primary feedback reduction and the associated throughput scaling law are rigorously derived in Section 6. Numerical results are presented in Section 7 followed by some concluding remarks in Section 8.

\subsection{Notations}

Here is a list of important notations used in this paper. $E$ [.] denotes the statistical expectation. $\operatorname{Pr}\{$.$\} represents proba-$ bility. The cumulative density function (CDF) of a random variable $Z$ is given by $F_{Z}(z)$ whereas $F_{Z}(z \mid Y)$ expresses the conditional CDF of $Z$ given $Y$. Let $X_{w}$ be a Bernoulli random variable such that $X_{w}=1$ with probability $w$ and $X_{w}=0$ with probability $1-w . \pi(i)$ denotes a permutation function from $\{1,2, \ldots, M\}$ to $\{1,2, \ldots, M\}$. $\Re_{+}$ represents the set of positive real numbers. $\frac{\partial y}{\partial x^{*}}$ denotes the partial derivative of $y$ with respect to $x$, evaluated at $x=x^{*} \cdot \min (a, b)=a$ if $a \leq b \cdot \mathbf{p}^{\mathrm{T}}$ represents the transpose of vector $\mathbf{p} . \mathcal{S}^{c}$ represents the complement of the set $\mathcal{S}$. We also use the notation $f(x)=O(g(x))$ to imply that $\limsup _{x \rightarrow \infty}\left|\frac{f(x)}{g(x)}\right|<\infty, f(x)=o(g(x))$ to imply that $\lim _{x \rightarrow \infty}\left|\frac{f(x)}{g(x)}\right|=0$ and $f(x)=\Theta(g(x))$ as $x \rightarrow \infty$ to imply that there exist positive constants $K_{1}$ and $K_{2}$ such that $K_{1}<\left|\frac{f(x)}{g(x)}\right|<K_{2}$.

\section{Related works}

For a single-user SISO cognitive underlay paradigm, POC was introduced in an ergodic capacity maximization problem [14], assuming that the delay-sensitive PU uses a constant power control (which is not energy-efficient from the primary user's perspective). However, this result cannot be generalized to downlink channels due to nonconvexity in the expression of ESC for a C-BC.

Information theoretic capacity notions for noncognitive SISO fading broadcast channels were investigated in, e.g. [5,7]. In [7], the authors showed that the base station allocates a given time slot (over which the fading channel remains invariant) to the user with the strongest reception only so as to maximize the total throughput, implying that an opportunistic scheduling or the so-called dynamic time-division-multiple-access (D-TDMA) is optimal. The amount of transmission power is allocated according to a water-filling policy. Using the duality result from [8], the authors of [9] investigated the optimal power control for ESC maximization in the SISO fading C-BC under both average/peak transmit power constraints and PIPC/AIPC, proving that opportunistic scheduling is the optimal scheme for achieving the ESC in C-BC. However, the optimal power allocation policy under POC (which is a more suitable metric to assure the quality of service for a delay-sensitive PU) has not been addressed so far in the existing literature.

Opportunistic user selection strategies have also motivated researchers to analyze how the ESC scales as the number of users $M$ increases. The analysis for throughput scaling in non-cognitive multiple-input multiple-output (MIMO) BC is provided in [15]. For underlay cognitive radio networks, there are a number of works studying multi-user diversity. For example, in [16], the authors studied the cognitive multiple-access (C-MAC) system under a peak transmit power constraint at each secondary transmitter and a peak interference power constraint at the primary receiver and analyzed capacity scaling as the ratio of the transmit power to the interference power approaches infinity. In [17], secondary capacity gains in C-MAC are investigated with the user selection criterion based on the strongest secondary direct channel and the weakest interference channel under a peak interference constraint. Later in [18], the multi-user interference diversity is examined for three types of cognitive networks, including C-MAC, $\mathrm{C}-\mathrm{BC}$, and cognitive parallel access channel (C-PAC), under peak transmit power and peak interference power constraints. Later in [19], the authors investigated ergodic sum capacity scaling in SISO C-MAC under a hybrid scheduling policy, i.e. by first selecting a set of eligible secondary transmitters which do not violate the interference quota and then choosing secondary transmitters that can be active. The same authors generalized the result in [19] to multi-user multi-antenna cognitive networks [20] with peak transmit power constraint at the secondary network, and peak interference power constraint at the primary receivers for both uplink and downlink channels was examined.

In order to make asymptotic analyses more tractable, the multi-user diversity has been studied mainly under suboptimal power allocation schemes, e.g. in [15,19-21]. Note that these results under suboptimal power allocation schemes simply provide lower bounds and do not necessarily reflect the optimal throughput scaling of the network. To establish a benchmark for justifying the efficiency of any suboptimal scheme, such as those based on partial CSI, it is crucial to derive throughput scaling under the optimal transmission power allocation scheme. 
Recently, the multi-user diversity gain under optimal power control in C-MAC under average transmit and average interference power constraints with various types of fading channels was investigated in [22].

\section{System model}

We consider a $\mathrm{C}-\mathrm{BC}$ with a secondary base station transmitting data to $M$ secondary receivers (SRs), sharing the same spectrum as a primary transmitter-primary receiver (PT-PR) pair. All terminals involved are equipped with a single antenna. Let $h_{i}, g, \alpha_{i}$, and $\beta$ denote the channel power gains from SBS to the $i$ th $\mathrm{SR}$, PT to PR, PT to the $i$ th SR, and SBS to PR, respectively, as illustrated in Figure 1. Let $\chi$ represent the joint channel state vector, i.e. $\chi=\left\{g, \beta, h_{1}, \ldots, h_{M}, \alpha_{1}, \ldots, \alpha_{M}\right\}$. All channel gains involved in this cognitive radio network are assumed to be mutually independent block-fading additive white Gaussian noise (BF-AWGN) channels with continuous CDFs [12]. Indeed, the BF-AWGN channel model has a long history of being used for both non-cognitive and cognitive radio networks. In particular, block-fading models have been used successfully in [23] for non-scalable video coding and data partitioning using H.264/AVC encoder in conversational applications, and a multi-resolution video/image data transmission system using hierarchical constellation was investigated in [24] under block-fading channel model. Authors in [25] analyzed the performance of multi-user multi-access wireless video communication in block-fading channel by using H.264/AVC encoder. In cognitive radio literatures, there is a great deal of literature assuming block-fading model [11,14,26-29]. In [29], the authors used the block-fading model for both primary and secondary channels. Typically, a primary transmitter disregards the existence of secondary networks and therefore adapts its transmission power based on a predefined power allocation policy which is not a function of the interference from the secondary networks (e.g. $[11,14])$. Hence, we assume that the PU allocates its transmission power based only on the direct gain $g$ between PT and PR, regardless of the interference from the secondary downlink channels [11]. The PU and the SBS are presumed to use random Gaussian codebook [11,14,30,31]. We also assume that a frequency division duplexing (FDD) scheme is implemented in both primary and secondary networks, i.e. CSI of primary and secondary direct channels is available at the corresponding transmitters via receiver feedback. In the first instance, SBS is assumed to have knowledge of perfect CSI on $\chi$ and primary user's power policy so that it also knows the primary user's power allocation policy for every realization of $\chi$.

Remark 1. Clearly, the knowledge of full CSI at SBS of the channels involving the primary terminals is not easy to achieve. In recent literature, some practical schemes have been suggested for obtaining such information at the ST in [32]. For ST-PR channels in FDD, it is suggested that the CSI at the PR is fed back to the SBS from the primary base station (PBS) (which is in communication with its PR) through an error-free backhaul link of negligible delay. Indeed, just as base station cooperation has become a reality via coordinated multi-point (CoMP) technology in the long-term evolution (LTE) systems, it is not unimaginable that the cooperation between SBS and PBS will become common, especially because it assists both the PUs and SUs in their interference management endeavour. It is also envisaged that some financial incentives may be used to encourage the primary service provider to facilitate such information exchange with the secondary service provider [33]. For PT-PR channels, various suggestions have been made including that of eavesdropping on PR feedback to PT [34] and receiving feedback from a cooperative SU node employed near the PR [35], while information about

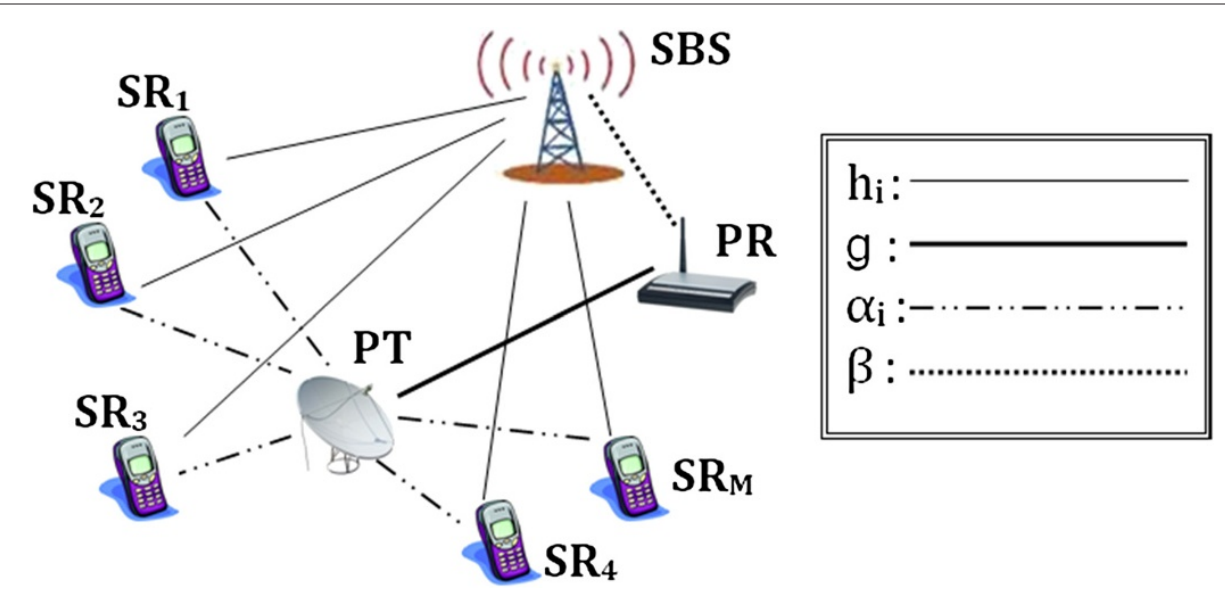

Figure 1 System model for C-BC. 
PT-SR and ST-SR channels can be obtained via classical channel feedback and training schemes based on pilot signals from the PT and ST, respectively. Finally, note that the analysis of throughput scaling in Section 5 based on full CSI serves the important purpose of a benchmark for any schemes based on imperfect CSI, such as the one with a 1-bit primary feedback scheme proposed in Section 6.

In this work, we assume that the QoS guarantee of the delay-sensitive PU with a target rate $r_{p}^{0}$ allows a maximum primary outage probability of $\epsilon_{p}$. In a typical wireless fading environment, even in the absence of interference from the secondary network, the PU may not be able to avoid an outage event when the PT-PR channel is in deep fade. For example, with an average or peak transmit power constraint, the PU cannot meet the target rate for a Rayleigh fading channel if it falls below a certain threshold [12]. We further presume that the PU's power policy is designed to allow for a maximum PU outage probability of $\epsilon_{p}^{0} \leq \epsilon_{p}$ in the absence of the secondary interference. Thus, the secondary network is allowed to cause an additional outage with probability $\epsilon_{p}-\epsilon_{p}^{0}$. Furthermore, let $P_{p}(g)$ define the transmission power policy at the PU. (As mentioned earlier, it is typical to assume that transmission power allocation policy at the PU is based on the direct gain $g$ only.).

Let $\mathbf{P}(\boldsymbol{\chi})=\left[P_{1}(\boldsymbol{\chi}), \ldots, P_{M}(\boldsymbol{\chi})\right]^{\mathrm{T}}$ be the mapping from $\Re_{+}^{2 M+2}$ to $\Re_{+}^{M}$, where $P_{i}(\chi)$ denote the SBS's transmit power allocated for the $i$ th SR. The PU's instantaneous rate expression can be written as

$$
r_{p}(\boldsymbol{\chi}, \mathbf{P}(\boldsymbol{\chi}))=\log \left(1+\frac{g P_{p}(g)}{\left(\beta \sum_{i=1}^{M} P_{i}(\boldsymbol{\chi})\right)+N_{0}}\right)
$$

The delay-sensitive primary network has an POC with a target rate $r_{p}^{0}$ and a maximum outage probability threshold $\epsilon_{p}$, such that

$$
\operatorname{Pr}\left\{r_{p}(\chi, \mathbf{P}(\chi))<r_{p}^{0}\right\} \leq \epsilon_{p}
$$

The power budget at the SBS can be either LTPC or STPC as shown in (3) and (4), respectively.

$$
\begin{aligned}
& E\left[\sum_{i=1}^{M} P_{i}(\boldsymbol{\chi})\right] \leq P_{\mathrm{av}} \\
& \sum_{i=1}^{M} P_{i}(\boldsymbol{\chi}) \leq P_{O}
\end{aligned}
$$

In this paper, we focus on solving the ergodic sum capacity maximizing problem in the secondary downlink subject to (2) and either (3) or (4). The maximum ergodic sum capacity achieved by the secondary network in this problem can be written as follows:

$$
\begin{aligned}
& C_{s}^{\mathrm{BC}}=\max _{\mathbf{P}(\chi) \in \mathcal{F}} E\left[r_{s}^{\mathrm{BC}}(\boldsymbol{\chi}, \mathbf{P}(\chi))\right] \\
& \text { s.t. (2) and either (3) or (4) }
\end{aligned}
$$

where $\mathcal{F}$ is the space of all mappings from $\Re_{+}^{2 M+2}$ to $\Re_{+}^{M}$. At each joint channel state $\chi$, the instantaneous sum capacity of secondary broadcast channels, $r_{s}^{\mathrm{BC}}(\chi, \mathbf{P}(\chi))$, is given by

$$
r_{s}^{\mathrm{BC}}(\boldsymbol{\chi}, \mathbf{P}(\boldsymbol{\chi}))=\sum_{i=1}^{M} \log \left(1+\frac{z_{\pi(i)} P_{\pi(i)}(\boldsymbol{\chi})}{1+z_{\pi(i)} \sum_{k=i+1}^{M} P_{\pi(k)}(\boldsymbol{\chi})}\right)
$$

where $\pi($.$) is the permutation that specifies the optimal$ decoding order, i.e. $z_{\pi(M)}>z_{\pi(M-1)}>\cdots>z_{\pi(1)}$ and $z_{i}=\frac{h_{i}}{\alpha P_{p}(g)+N_{0}}$. In other words, $z_{i}$ represents the signalto-interference-plus-noise ratio (SINR) of the $i$ th user whereas $z_{\pi(i)}$ is the SINR of the $\pi(i)$-th user based on the optimal decoding order $\pi(i)$. Note that the sum-capacity expression can be obtained by successive decoding with interference cancellation $[8,36]$. Also, note that we drop the constant $\frac{1}{2}$ in the instantaneous rate expressions in (6) and use natural logarithm for simplicity. We also assume that the AWGN variance in each SR is given by $N_{0}$. Obviously, $r_{s}^{\mathrm{BC}}(\chi, \mathbf{P}(\chi))$ is not concave over $\mathbf{P}(\chi)$. In the next section, we first establish the optimality of an opportunistic scheduling structure in our setup which allows us to reformulate (5) as a convex optimization problem.

\section{Optimal power strategies}

In this section, we focus on the optimal power allocation strategy in the secondary network which maximizes ESC under POC at the primary link with a limited power budget at the SBS. The transmission power resource is confined by either LTPC (average transmit power constraint) or STPC (peak transmit power constraint). First, we look at the ESC maximizing problem subject to POC when the transmission power at the SBS is limited by LTPC in Section 4.1. Later, the ESC maximizing problem under POC and STPC will be considered in Section 4.2.

\subsection{Optimal power policy for LTPC}

The secondary ergodic sum capacity maximization problem under POC and LTPC is defined as follows:

$$
C_{s}^{\mathrm{BC}}=\max _{\mathbf{P}(\boldsymbol{\chi})} . E\left[r_{s}^{\mathrm{BC}}(\boldsymbol{\chi}, \mathbf{P}(\boldsymbol{\chi}))\right] \text { s.t. (2) and (3) }
$$

The optimal power policy solving (7) is given by Theorem 1. 
Theorem 1. The optimal power control solution for (7) is $\mathbf{p}^{*}(\boldsymbol{\chi})=X_{w^{*}}(\boldsymbol{\chi}) \mathbf{p}_{1}^{*}(\boldsymbol{\chi})+\left(1-X_{w^{*}}(\boldsymbol{\chi})\right) \mathbf{p}_{2}^{*}(\boldsymbol{\chi}), \mathbf{p}_{1}^{*}(\boldsymbol{\chi}), \mathbf{p}_{2}^{*}(\boldsymbol{\chi})$ and $w^{*}(\chi)$ are defined as follows:

$$
p_{1, i}^{*}(\chi)= \begin{cases}\min \left(p_{W F}^{*}(\chi), p_{R P}^{*}(\chi)\right), & w^{*}(\chi)=1, i=i^{*} \\ 0, & \text { otherwise }\end{cases}
$$

$$
\begin{aligned}
& p_{2, i}^{*}(\chi)= \begin{cases}p_{W F}^{*}(\chi), & w^{*}(\chi)=0, i=i^{*} \\
0, & \text { otherwise }\end{cases} \\
& w^{*}(\chi)=\left\{\begin{array}{l}
1, \mathscr{B}_{1, \chi}^{B}>\mathscr{B}_{2, \chi}^{B} \\
0, \mathscr{B}_{1, \chi}^{B}<\mathscr{B}_{2, \chi}^{B}
\end{array}\right.
\end{aligned}
$$

where $i^{*}=\arg \max _{m \in \mathcal{I}} z_{m}, p_{W F}^{*}(\boldsymbol{\chi})=\left(\frac{1}{\Lambda^{*}}-\frac{1}{\max z_{m}}\right)^{+}$, $p_{R P}^{*}(\boldsymbol{\chi})=\frac{1}{\beta}\left(\frac{g P_{p}(g)}{\exp \left(r_{p}^{0}\right)-1}-N_{0}\right)^{+}, \mathscr{B}_{1, \boldsymbol{\chi}}^{B}=r_{s}^{B C}\left(\boldsymbol{\chi}, \mathbf{p}_{1}^{*}(\boldsymbol{\chi})\right)-$ $\Lambda^{*} \mathbf{1}^{T} \mathbf{p}_{1}^{*}(\boldsymbol{\chi})+S^{*}$ and $\mathscr{B}_{2, \chi}^{B}=r_{s}^{B C}\left(\chi, \mathbf{p}_{2}^{*}(\chi)\right)-\Lambda^{*} \mathbf{1}^{T} \mathbf{p}_{2}^{*}(\chi)$.

The Lagrange multipliers $\Lambda^{*}$ and $S^{*}$ are the solutions to $E\left[\sum_{i=1}^{M} P_{i}^{*}(\chi)\right]=P_{a v}$ and $E\left[w^{*}(\chi)\right] \geq 1-\epsilon_{p}$.

Proof. The proof relies on the optimality of an opportunistic scheduling structure as first proved in Appendix 1 (see Lemmas 1 and 2). Based on this result, the proof of the optimal power control policy as presented by Theorem 1 above is given in Appendix 2.

Theorem 1 implies that the optimal power control policy of the problem (7) possesses an opportunistic scheduling structure in each joint channel state $\chi$, i.e. the SBS allocates non-negative power, say $\breve{P}(\chi)$, to only the $i$ th SR with $i=\arg \max _{m \in \mathcal{I}} z_{m}$ and zero power to other remaining SRs. Although $\breve{P}(\boldsymbol{\chi})$ does not explicitly depend on the index of each $i$ th secondary receiver, it intrinsically represents the power allocated to the scheduled user in each specific joint channel state $\chi$. For simplicity, define $z_{\max }=$ $\max _{m} z_{m}$. By the opportunistic scheduling structure, the original non-convex problem in (7) can be converted to (11) as follows:

$$
\begin{aligned}
& \max _{\breve{P}(\boldsymbol{\chi}) \geq 0} E\left[r_{s}(\boldsymbol{\chi}, \breve{P}(\chi))\right] \\
& \text { s.t. } \operatorname{Pr}\left\{\log \left(1+\frac{g P_{p}(g)}{N_{0}+\beta \breve{P}(\boldsymbol{\chi})}\right)<r_{p}^{0}\right\} \leq \epsilon_{p}, E[\breve{P}(\boldsymbol{\chi})] \leq P_{\mathrm{av}}
\end{aligned}
$$

where $r_{s}(\chi, \breve{P}(\chi))=\log \left(1+\breve{P}(\chi) z_{\max }\right)$ represents the instantaneous sum-capacity expression of the secondary broadcast channels by using the opportunistic scheduling structure, and the objective function in (11) is concave over $\breve{P}(\chi)$.
Theorem 1 also reveals that the optimal power policy of the problem (7) can be constructed by utilizing two deterministic power allocation schemes with opportunistic scheduling structure, i.e. $\mathbf{P}^{*}(\boldsymbol{\chi})=\mathbf{p}_{1}^{*}(\boldsymbol{\chi})$ in (8) if $w^{*}(\boldsymbol{\chi})=1$ and $\mathbf{p}_{2}^{*}(\boldsymbol{\chi})$ in (9) if $w^{*}(\boldsymbol{\chi})=0$. The intuitive explanation of the optimal power control policy is that for a given joint channel state $\chi$, the SBS can design the power control policy under only two options, i.e. by either putting the PU in outage or not putting the PU not in outage. The deterministic power policy $\mathbf{p}_{2}^{*}(\boldsymbol{\chi})$ in (9) represents the optimal power policy which the PU is in outage, i.e. the SBS allocates power $p_{2, i}^{*}(\boldsymbol{\chi})=p_{\mathrm{WF}}^{*}(\boldsymbol{\chi})$ for the $i$ th SR with $i=\arg \max _{m \in \mathcal{I}} z_{m}$ according to the wellknown water-filling power policy and zero power to the other remaining SRs. Note that the solution is similar to the optimal power policy of the sum capacity maximizing problem in non-cognitive broadcast channels [7] since the SBS decides to put the PU in outage. On the other hand, $\mathbf{p}_{1}^{*}(\boldsymbol{\chi})$ denotes the optimal power policy when the SBS ensures the service quality in the primary link. In this case, the SBS still allocates a transmission power with water-filling power policy to the scheduled user as long as $p_{\mathrm{WF}}^{*}(\boldsymbol{\chi}) \leq p_{\mathrm{RP}}^{*}(\boldsymbol{\chi})=$. However, if $p_{\mathrm{WF}}^{*}(\boldsymbol{\chi})>p_{\mathrm{RP}}^{*}(\boldsymbol{\chi})$, the transmission power is $p_{\mathrm{RP}}^{*}(\chi)$ since $p_{\mathrm{RP}}^{*}(\chi)$ is the maximum transmission power from the SBS that can ensure that the PU is not in outage, i.e. the primary rate equals the primary target rate. Therefore, the water-filling power policy is capped by $p_{\mathrm{RP}}^{*}(\boldsymbol{\chi})$. Between these two deterministic power policies, the SBS utilizes the policy which returns the highest benefit, i.e. if the benefit function $\mathscr{B}_{1, \chi}^{B}$ of the power policy $\mathbf{p}_{1}^{*}(\boldsymbol{\chi})$ is greater than the benefit function $\mathscr{B}_{2, \chi}^{B}$ of the power policy $\mathbf{p}_{2}^{*}(\chi)$, then $w^{*}(\chi)=1$. If $\mathscr{B}_{1, \chi}^{B}<\mathscr{B}_{2, \chi}^{B}, w^{*}(\chi)=0$, implying that the SBS uses the power policy $\mathbf{p}_{2}^{*}(\chi)$.

\subsection{Optimal power policy for STPC}

The secondary ergodic sum capacity maximization problem under POC and STPC is defined as follows:

$$
C_{s}^{\mathrm{BC}}=\max _{\mathbf{P}(\boldsymbol{\chi})} . E\left[r_{s}^{\mathrm{BC}}(\boldsymbol{\chi}, \mathbf{P}(\boldsymbol{\chi}))\right] \text { s.t. (2) and (4) }
$$

The optimal power policy solving (12) is given by Theorem 2. See Appendix 3 for a detailed proof.

Theorem 2. The optimal power control solution for (12) is $\mathbf{P}^{*, s t}(\boldsymbol{\chi})=X_{w_{1}^{*, s t}}(\boldsymbol{\chi}) \mathbf{p}_{1}^{*, s t}(\boldsymbol{\chi})+\left(1-X_{w_{1}^{*, s t}}(\boldsymbol{\chi})\right) \mathbf{p}_{2}^{*, s t}(\boldsymbol{\chi})$, where $\mathbf{p}_{1}^{*, s t}(\boldsymbol{\chi}), \mathbf{p}_{2}^{*, s t}(\boldsymbol{\chi})$ and $w^{*, s t}(\boldsymbol{\chi})$ are defined as follows:

$$
p_{1 i}^{* s t}(\chi)= \begin{cases}\min \left(P_{O}, p_{R P}^{*}(\chi)\right), & w^{*, s t}(\chi)=1, i=i^{*} \\ 0, & \text { otherwise }\end{cases}
$$




$$
\begin{aligned}
& p_{2 i}^{*, s t}(\boldsymbol{\chi})= \begin{cases}P_{O}, & w^{*, s t}(\boldsymbol{\chi})=0, i=i^{*} \\
0, & \text { otherwise }\end{cases} \\
& w^{*, s t}(\boldsymbol{\chi})=\left\{\begin{array}{l}
1, \mathscr{B}_{1, \boldsymbol{\chi}}^{B, s t}>\mathscr{B}_{2, \boldsymbol{\chi}}^{B, s t} \\
0, \mathscr{B}_{1, \boldsymbol{\chi}}^{B, s t}<\mathscr{B}_{2, \boldsymbol{\chi}}^{B, s t}
\end{array}\right.
\end{aligned}
$$

where 'st' in the superscript stands for 'short-term' and $i^{*}=\arg \max _{m \in \mathcal{I}} z_{m}$. Also, $\mathscr{B}_{1, \boldsymbol{\chi}}^{B, s t}=r_{s}\left(\boldsymbol{\chi}, \mathbf{p}_{1}^{*, s t}(\boldsymbol{\chi})\right)-$ $\Lambda^{*, s t}(\boldsymbol{\chi}) \mathbf{1}^{T} \mathbf{p}_{1}^{*, s t}(\boldsymbol{\chi})+S^{*, s t}$ and $\mathscr{B}_{2, \boldsymbol{\chi}}^{B, s t}=r_{s}\left(\boldsymbol{\chi}, \mathbf{p}_{2}^{*, s t}(\boldsymbol{\chi})\right)-$ $\Lambda^{*, s t}(\boldsymbol{\chi}) \mathbf{1}^{T} \mathbf{p}_{2}^{*, s t}(\chi)$. Lagrange multipliers $\Lambda^{*, s t}(\boldsymbol{\chi})$ and $S^{*, s t}$ are the solutions to $\sum_{i=1}^{M} P_{i}^{*, s t}(\chi) \leq P_{O}$ and $E\left[w^{*, s t}(\chi)\right] \geq$ $1-\epsilon_{p}$

Similar to Theorem 1 in LTPC case, Theorem 2 implies that an opportunistic scheduling structure is optimal in each joint channel state $\chi$ for which the SBS schedules the SR with the maximum $z_{i}$ only. Thus, the original nonconvex problem in (12) can be converted to (16) as follows:

$$
\begin{aligned}
& \max _{\breve{P}^{\mathrm{st}}(\boldsymbol{\chi}) \geq 0} E\left[r_{s}\left(\boldsymbol{\chi}, \breve{P}^{\mathrm{st}}(\boldsymbol{\chi})\right)\right] \\
& \text { s.t. } \quad \operatorname{Pr}\left\{\log \left(1+\frac{g P_{p}(g)}{N_{0}+\beta \breve{P}^{\mathrm{st}}(\boldsymbol{\chi})}\right)<r_{p}^{0}\right\} \leq \epsilon_{p}, \breve{P}^{\mathrm{st}}(\boldsymbol{\chi}) \leq P_{O} .
\end{aligned}
$$

Similar to Section 4.1, it can be shown that the objective function in (16) is concave over $\breve{P}^{\text {st }}(\chi)$.

For the STPC, Theorem 2 reveals that the SBS also chooses one of the two deterministic power allocation policies, i.e. the SBS can either decide to ensure the QoS in the primary link by using the power policy $\mathbf{p}_{1}^{* \text {,st }}(\boldsymbol{\chi})$ in (14) or ignore the PU's QoS requirement by using the power policy $\mathbf{p}_{2}^{* \text {,st }}(\boldsymbol{\chi})$ in (14). This decision relies on $w^{*, \text { st }}(\boldsymbol{\chi})$ in (15). However, the SBS allocates transmission power based on the constant power control with peak power $P_{O}$ to the scheduled SR in the STPC case instead of using the water-filling power policy in the LTPC case.

Remark 2. As opposed to the result in [14] where the PU utilizes a constant power policy, the optimal power allocation strategies in Theorems 1 and 2 are applicable to any arbitrary PU power allocation policy as long as the primary user is oblivious to the secondary network, i.e. the PU's power allocation policy is not a function of the SBS's transmission power allocation function.

\section{Throughput scaling with truncated channel inversion policy at $\mathrm{PU}$}

In this section, we derive asymptotic scaling laws of the SBS ergodic sum capacity when the number of SUs grows large, under the assumption that all channel power gains are independent and identically distributed and they are also presumed to be exponentially distributed with unity mean. Also, we focus on the case where the PU uses the TCI policy. The motivation behind the assumption of a TCI power policy at the PU is that TCI is the optimal power allocation policy for minimizing the information outage probability under a long/short transmit power constraint for a delay-sensitive user. More specifically, the TCI power control policy employed at the PU in this paper can be described as follows: PU's transmit power adapts according to the direct channel gain $g$ when the PU is ON with transmission power $P_{p}(g)=\frac{\left(e^{r_{p}^{0}}-1\right) N_{0}}{g}$ when $g \geq g_{T}$ and $P_{p}(g)=0$ otherwise. Note also that $g_{T}$ is chosen such that for $\operatorname{Pr}\left(g<g_{T}\right)=\epsilon_{p}^{0}$.

Given that the PU employs a TCI power policy, in Section 5.1, we present the optimal channel partitioning structures dictated by the optimal power allocation strategies in Theorem 1 for LTPC and in Theorem 2 for STPC. We show that the space of joint channel states $\chi \in \Re_{+}^{2 M+2}$ can be separated into three regions for the ESC maximizing problem subject to POC and either LTPC or STPC. Aided by these results in Section 5.1, the optimal throughput scaling laws of the SBS ergodic sum capacity under POC and LTPC will be derived in Section 5.2. Finally, Section 5.3 will present the optimal throughput scaling laws of the SBS ergodic sum capacity under POC and STPC.

\subsection{Optimal channel state partitioning structure}

Assuming a TCI policy at the PU, the space of joint channel states $\chi \in \Re_{+}^{2 M+2}$ can be divided into three regions when the SBS employs the optimal power allocation policy. First, we summarize the details of the three channel state regions for LTPC in Table 1 together with the power allocation policy, outage status at the PU, and the probability of each channel state region.

Table 1 reveals the intuitive explanation of the transmission mechanism at the SBS for the LTPC case. In region 1 , the PU stops transmitting as $g<g_{T}$ and faces an outage even if there is no interference from the secondary network. In this case, the SBS will allocate power based

Table 1 Three possible channel state regions for the fading channel state $\chi$ for LTPC

\begin{tabular}{ccccc}
\hline Region & $\begin{array}{c}\text { Channel } \\
\text { state } \\
\text { region }\end{array}$ & $\begin{array}{c}\text { Optimal } \\
\text { power } \\
\text { control }\end{array}$ & $\begin{array}{c}\text { Outage } \\
\text { at PU }\end{array}$ & Probability \\
\hline 1 & $g<g_{T}$ & $p_{\mathrm{WF}}^{*}$ & Yes (PU turns OFF) & $\epsilon_{p}^{0}$ \\
2 & $g \geq g_{T,}, \mathscr{B}_{1, \boldsymbol{\chi}}^{B} \leq \mathscr{B}_{2, \boldsymbol{\chi}}^{B}$ & $p_{\mathrm{WF}}^{*}$ & Yes & $\epsilon_{p}-\epsilon_{p}^{0}$ \\
3 & $g \geq g_{T,}, \mathscr{B}_{1, \boldsymbol{\chi}}^{B}>\mathscr{B}_{2, \boldsymbol{\chi}}^{B}$ & $p_{\mathrm{RP}}^{*}=0$ & No & $1-\epsilon_{p}$
\end{tabular}


on water-filling power policy to the scheduled SR. Also note that there is no interference from the primary transmitter to the secondary network as the PU turns off in this case. When $g \geq g_{T}$, the PU is ON with $P_{p}(g)=\frac{k_{1}}{g}$ where $k_{1}=\left(e^{r_{p}^{0}}-1\right) N_{0}$ and thus, any positive transmission power from the SBS can put the PU in outage. Therefore, the SBS must switch OFF if the SBS decides to protect the service quality in the primary link. In region 2, the SBS still decides to transmit because the power policy that puts PU in outage $\left(\mathbf{p}_{2}^{*}\right)$ returns a higher profit than the power policy that protects the primary transmission $\left(\mathbf{p}_{1}^{*}\right)$, i.e. $\mathscr{B}_{1, \chi}^{B} \leq \mathscr{B}_{2, \chi}^{B}$. The SBS then allocates power $p_{\mathrm{WF}}^{*}$ to the scheduled SR. In region 3, the SBS knows that it is more profitable to stop its data transmission and ensure the service quality in the PU's link, i.e. $\mathscr{B}_{1, \chi}^{B}>\mathscr{B}_{2, \chi}^{B}$.

In the ESC maximizing problem subject to POC and STPC, the optimal power control strategy according to Theorem 2 can be utilized to separate the space of joint channel states $\chi \in \mathfrak{R}_{+}^{2 M+2}$ into three distinct channel state regions as summarized in Table 2 . Also note that the interpretation from Table 2 is quite similar to Table 1, except for using a constant power control at the peak power $P_{O}$ rather than the water-filling power policy.

Remark 3. Note that if $S^{*}=0$ in the LTPC case (or $S^{* \text {,st }}=0$ in the STPC case), the SBS can transmit with $p_{\mathrm{WF}}^{*}$ (water-filling) in the LTPC case or $P_{O}$ in the STPC case without making the $\mathrm{POC}$ active, as if the primary network never existed. This scenario then simplifies to a non-cognitive broadcast channel, and it has been shown in [15] that in this case the ergodic sum rate scales according to $\log (\log M)$ as $M$ becomes large. In this paper, we will analyze the throughput scaling laws only for the case when $S^{*}>0$ for LTPC and $S^{*, \text { st }}>0$ for STPC.

\subsection{Throughput scaling for the LTPC case}

In this section, we focus on the derivation of throughput scaling when the SBS employs the optimal power allocation policy according to Theorem 1 (LTPC case). The

Table 2 Three possible channel state regions for the fading channel state $\chi$ for STPC

\begin{tabular}{ccccc}
\hline Region & $\begin{array}{c}\text { Channel } \\
\text { state } \\
\text { region }\end{array}$ & $\begin{array}{c}\text { Optimal } \\
\text { power } \\
\text { control }\end{array}$ & $\begin{array}{c}\text { Outage } \\
\text { at PU }\end{array}$ & Probability \\
& & & \\
\hline
\end{tabular}

$\begin{array}{llccc}1 & g<g_{T} & P_{O} & \text { Yes (PU turns OFF) } & \epsilon_{p}^{0} \\ 2 & g \geq g_{T}, \mathscr{B}_{1, \boldsymbol{\chi}}^{B, s t} \leq \mathscr{B}_{2, \boldsymbol{\chi}}^{B, s t} & P_{O} & \text { Yes } & \epsilon_{p}-\epsilon_{p}^{0} \\ 3 & g \geq g_{T}, \mathscr{B}_{1, \boldsymbol{\chi}}^{B, s t}>\mathscr{B}_{2, \boldsymbol{\chi}}^{B, s t} & p_{\mathrm{RP}}^{*}=0 & \text { No } & 1-\epsilon_{p}\end{array}$

main result of this section is summarized in Theorem 3 , a detailed proof of which is provided in Appendix 4.

Theorem 3. When PU uses TCI power control policy, the asymptotic scaling law of the SBS ergodic sum capacity under a POC and a LTPC with optimal power control is given by

$$
\lim _{M \rightarrow \infty} \frac{C_{s}^{B C *}}{\log (\log M)}=\epsilon_{p}
$$

The intuitive explanation for Theorem 3 is that when PU uses a TCI power control, the SBS is forced to turn off for the channel fading states in which the PU link QoS can be compromised since the SBS transmission results in the violation of POC. Since the primary outage probability threshold is $\epsilon_{p}$, the SBS is allowed to transmit with probability $\epsilon_{p}$ which leads to the pre-log factor term.

\subsection{Throughput scaling for the STPC case}

In this section, we will derive the throughput scaling result due to the optimal power allocation in Theorem 2 (STPC). The main result of this section can be summarized in Theorem 4, a detailed proof of which is provided in Appendix 5.

Theorem 4. When PU uses the TCI power control policy, the asymptotic scaling law of the SBS ergodic sum capacity under a POC and a STPC with optimal power control is given by

$$
\lim _{M \rightarrow \infty} \frac{C_{s}^{\mathrm{BC} *}}{\log (\log M)}=\epsilon_{p}
$$

The intuitive reason for the appearance of the pre-log factor $\epsilon_{p}$ is similar to the LTPC case with TCI power control at PU.

\section{Suboptimal transmission scheme with feedback reduction}

In the optimal power policy, the SBS requires the information of the entire channel state $\chi$ which includes $g$ and $\beta$ in order to schedule the SR as well as compute the optimal outage region of the PU and the optimal transmission power. As mentioned earlier, the SBS can obtain the perfect CSI of the interference gain $\beta$ between the SBS and the primary receiver by measuring the received power of signals transmitted by the PR. For PT-PR channels, it is suggested that the SBS can eavesdrop on PR feedback to PT [34] or receive the feedback from a cooperative SU node employed near the PR [35]. Both ST-PR and PT-PR link information can also be obtained via backhaul links from a cooperative PBS possibly via pricing [33]. In this section, we propose a new suboptimal scheme which can further reduce the cooperation between the 
primary receiver and the secondary network by receiving just only 1-bit feedback from the PR, or if the SBS can feedback from PR, no cooperation is required. In this scheme, the PU predefines the PU outage region (suboptimal) based on the direct gain $g$ only, so the outage/nonoutage status of the PU can be realized at the SBS by using just 1-bit feedback from the PR. The corresponding opportunistic scheduling and power allocation policy for either LTPC or STPC are also proposed. It is important to note that by using this scheme, the SBS ESC still scales like $\epsilon_{p} \log (\log M)$ as $M$ grows large (asymptotically optimal). The details of the suboptimal scheme are as follows:

- Initially, the channel state for the direct channel gain $g$ between the PT and the PR is divided into two regions: the non-outage region $g \in\left[g_{T}, \tilde{g}_{T}\right]$, where the secondary network must not transmit in order to guarantee the primary link's service quality, and the outage region, $g \in \mathscr{G}_{\text {outage }}=\left[0, g_{T}\right] \bigcup\left[\tilde{g}_{T}, \infty\right)$, where the secondary network can transmit. The value $\tilde{g}_{T}$ is chosen such that $\epsilon_{p}-\epsilon_{p}^{0}=1-F_{G}\left(\tilde{g}_{T}\right)$, so that the outage probability in the primary link is ensured to be less than $\epsilon_{p}$. Further note that $g_{T}$ and $\tilde{g}_{T}$ depend on the distribution of the channel gain $g$ rather than their instantaneous values.

- Feedback policy The primary receiver sends a feedback of ' 0 ' to its transmitter (and the SBS if cooperating) if $g \notin \mathscr{G}_{\text {outage }}$ to halt the transmission activity in the secondary network, whereas it sends a feedback of ' 1 ' if the SBS is allowed to transmit when $g \in \mathscr{G}_{\text {outage. }}$

- Opportunistic scheduling policy In the perfect CSI case, the SBS will schedule the SR with maximum $\frac{h_{m}}{\alpha_{m} P_{p}(g)+N_{0}}$ which requires the knowledge of $P_{p}(g)$. However, in the new scheme, we can assume that the SBS is pessimistic by always assuming that $P_{p}(g)=P_{p}\left(\tilde{g}_{T}\right)$, i.e. the PU transmits the maximum power of the range $g \in \mathscr{G}_{\text {outage. }}$. Therefore, the SBS will schedule the SR with maximum $\tilde{\rho}_{m}=\frac{h_{m}}{\alpha_{m} P_{p}\left(\tilde{g}_{T}\right)+N_{0}}$ and the ergodic sum rate can be expressed as

$C_{\text {Partial }}^{\mathrm{BC}}=\operatorname{Pr}\left\{\mathscr{G}_{\text {outage }}\right\} E\left[\log \left(1+P_{\text {Partial }}(\chi) \tilde{\rho}_{\max }\right) \mid \mathscr{G}_{\text {outage }}\right]$

where $\tilde{\rho}_{\max }=\max _{m} \tilde{\rho}_{m}$ and $P_{\text {Partial }}(\boldsymbol{\chi})$ is the SBS's transmission power allocated to the SR with maximum $\tilde{\rho}_{\max }$. Note that the statistical behaviour of $\tilde{\rho}_{m}$ is independent of $g$.

- Power allocation policies and throughput scaling results For STPC, the power allocation policy at the SBS is straightforward. The SBS transmits with the maximum power $P_{O}$ when $g \in \mathscr{G}_{\text {outage. }}$. The ergodic sum rate for STPC becomes

$$
\begin{aligned}
C_{\text {Partial }}^{\text {BC, STPC }} & =\operatorname{Pr}\left\{\mathscr{G}_{\text {outage }}\right\} E\left[\log \left(1+P_{O} \tilde{\rho}_{\text {max }}\right) \mid \mathscr{G}_{\text {outage }}\right] \\
& =\epsilon_{p} E\left[\log \left(1+P_{O} \tilde{\rho}_{\text {max }}\right)\right]
\end{aligned}
$$

For LTPC, we have to solve the following optimization problem:

$$
\begin{aligned}
& \underset{P_{\text {Partial }}(\chi)}{\max } \operatorname{Pr}\left\{\mathscr{G}_{\text {outage }}\right\} \cdot E\left[\log \left(1+P_{\text {Partial }}(\boldsymbol{\chi}) \tilde{\rho}_{\text {max }}\right) \mid \mathscr{G}_{\text {outage }}\right] \\
& \text { s.t. } \operatorname{Pr}\left\{\mathscr{G}_{\text {outage }}\right\} E\left[P_{\text {Partial }}(\boldsymbol{\chi}) \mid \mathscr{G}_{\text {outage }}\right] \leq P_{\text {av }} .
\end{aligned}
$$

It is easy to show that the solution to the optimization problem in (19) is still water-filling, i.e. $P_{\text {Partial }}(\chi)=\left(\frac{1}{\Lambda_{\text {Partial }}}-\frac{1}{\tilde{\rho}_{\max }}\right)^{+}$for $g \in \mathscr{G}_{\text {outage }}$ and $P_{\text {Partial }}(\boldsymbol{\chi})=0$ for otherwise. Note that $\Lambda_{\text {Partial }}$ satisfies LTPC with equality.

Therefore, the ergodic sum rate for LTPC becomes

$$
\begin{aligned}
C_{\text {Partial }}^{\mathrm{BC}, \mathrm{LTPC}} & =\operatorname{Pr}\left\{\mathscr{G}_{\text {outage }}\right\} \cdot E\left[\log \left(\frac{\tilde{\rho}_{\max }}{\Lambda_{\text {Partial }}}\right) 1_{\left\{\Lambda_{\geq \text {Partial }} \tilde{\rho}_{\text {max }}\right\}} \mid \mathscr{G}_{\text {outage }}\right] \\
& =\epsilon_{p} E\left[\log \left(\frac{\tilde{\rho}_{\max }}{\Lambda_{\text {Partial }}}\right) 1_{\left\{\tilde{\rho}_{\max } \geq \Lambda_{\text {Partial }}\right\}}\right]
\end{aligned}
$$

It is easy to show that both $E\left[\log \left(1+P_{O} \tilde{\rho}_{\max }\right)\right]$ in the STPC case and $E\left[\log \left(\frac{\tilde{\rho}_{\max }}{\Lambda_{\text {Partial }}}\right) 1_{\left\{\tilde{\rho}_{\max } \geq \Lambda_{\text {Partial }}\right\}}\right]$ in the LTPC case scale according to $\log (\log M)$.

Therefore, the ergodic sum capacity scales according to $\epsilon_{p} \log \log M$ for LTPC as well as STPC under this suboptimal scheme.

\section{Numerical results}

In this section, we present some numerical results on the performance of the proposed optimal power policies for the capacity maximization problem with POC and LTPC or STPC. All channel gains involved are assumed to be Rayleigh fading, and the corresponding channel power gains are taken to be exponentially distributed with unit mean. Noises at PR and all SRs are presumed to be equal and AWGN with unit variance, i.e. $N_{0}=1$. Note that this allows the transmit power at the primary and secondary transmitters to be interpreted as signal-to-noise ratio at the transmitter side. Unless specified otherwise, PU's target rate is $r_{p}^{0}=1.25 \mathrm{nat} / \mathrm{channel}$ use and the primary outage probability threshold $\epsilon_{p}=0.1$. For the primary TCI power policy, we set $\epsilon_{p}^{0}=0.05$. For convenience, we further assume that all secondary receivers are identical. The simulation results are based on a Monte Carlo method averaged over $10^{5}$ channel realizations. 
7.1 The effect of POC on the ergodic sum capacity of C-BC In this section, we observe the effect of POC on the SBS downlink sum capacity when PU uses a TCI power policy through Figure 2 in both LTPC and STPC cases. For a fixed number of secondary receivers $M=4$, Figure 2 shows that with a decreasing PU's outage probability threshold for a fixed target rate, SBS downlink sum capacity decreases for both LTPC and STPC when PU applies the TCI power strategy, as expected. The same feature is also noticed when PU's outage probability threshold is fixed with an increasing rate. The results are due to the fact that the POC becomes stricter when the PU's outage probability threshold is reduced or the target rate is increased.

\subsection{Throughput scaling results with $\mathrm{TCl}$ power policy at the PU}

In this part, we will discuss how the SBS ergodic sum capacity behaves as the number of secondary receivers grows under the optimal power policy in Theorem 2 for STPC. In this problem, there are also a number of parameters which affect the SBS sum ergodic capacity from the optimal power policy in Theorem 2 for STPC, including $\epsilon_{p}^{0}$, the PU's target rate $r_{p}^{0}$, PU's outage probability constraint $\epsilon_{p}$, and the secondary network's power budget ( $P_{O}$ for STPC). The results for the LTPC case are similar and excluded to save space. From Figure 3, a decrease in the secondary power budget significantly reduces the SBS sum throughput i.e. when $P_{O}$ is decreased, the throughput scaling is shifted downwards. Next, a decrease in $\epsilon_{p}^{0}$ makes the sum throughput drop because the truncated threshold $g_{T}=-\log \left(1-\epsilon_{p}^{0}\right.$ ) (for the Rayleigh fading model) is also reduced. Although the additional outage caused by the secondary network is increased, SBS stops transmitting if it decides to protect the PU when the PU is active in the TCI case. Further, suppose that $\epsilon_{p, 1}^{0}>\epsilon_{p, 2}^{0}$, then $g_{T, 1}>g_{T, 2}$. With $\epsilon_{p}^{0}=\epsilon_{p, 2}^{0}$, SBS can transmit when $g \in\left[g_{T, 2}, g_{T, 1}\right]$. However, the interference from primary transmitter to the secondary receivers is high in this additional range as the PU transmits with power $\frac{\left(e^{r^{0}}-1\right) N_{0}}{g}$. This implies that the range $g \in\left[g_{T, 2}, g_{T, 1}\right]$ does not help enhance the SBS sum throughput, resulting in a decline in the sum throughput as $\epsilon_{p}^{0}$ is reduced. Finally, $r_{p}^{0}$ affects the throughput scaling via the POC as shown in Figure 3. As $r_{p}^{0}$ is increased to 4.00 nat/channel use, the POC becomes stricter, thereby resulting in a decline in the SBS sum throughput.

Next, we present the results for the normalized throughput $\frac{C_{s}^{\mathrm{BC}}}{\log (\log M)}$ as a function of the number of secondary receivers $M$ for both LTPC and STPC, when the PU employs the TCI power policy. These simulations illustrate the throughput scaling results of Theorems 3 and 4. Also, the normalized throughput results due to the proposed suboptimal transmission scheme with feedback reduction are illustrated. For these simulations, $P_{\mathrm{av}}$ for the LTPC case and $P_{O}$ for the STPC case are set to $1 \mathrm{~dB}$. The results in Figure 4 clearly illustrate that the normalized throughput converges to $\epsilon_{p}$ as $M$ becomes larger and larger. The results in Figure 4 also confirm that 1-bit feedback from the PU is effective enough to ensure that the normalized throughput of the proposed scheme asymptotically converges to $\epsilon_{p}$ as $M$ becomes large.

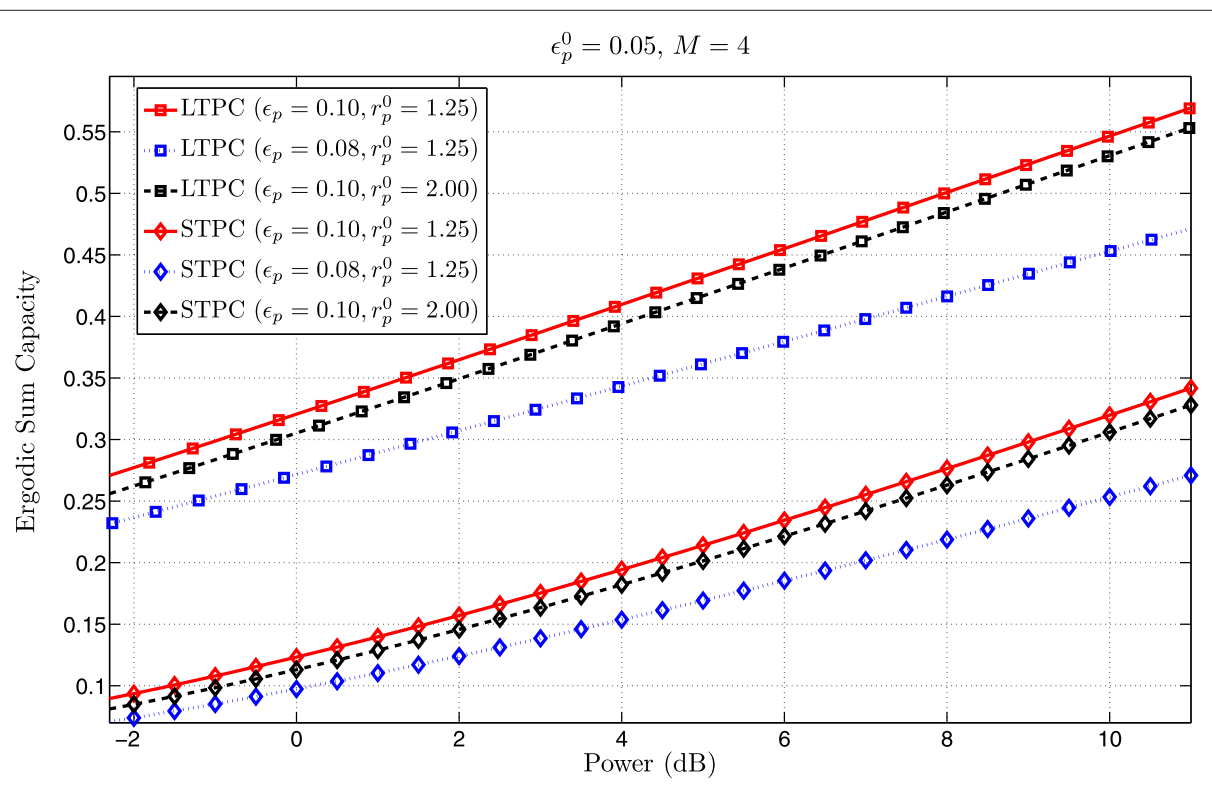

Figure 2 SBS ergodic sum capacity against power budget $\epsilon_{p}^{0}=0.05$ and $M=4$. 


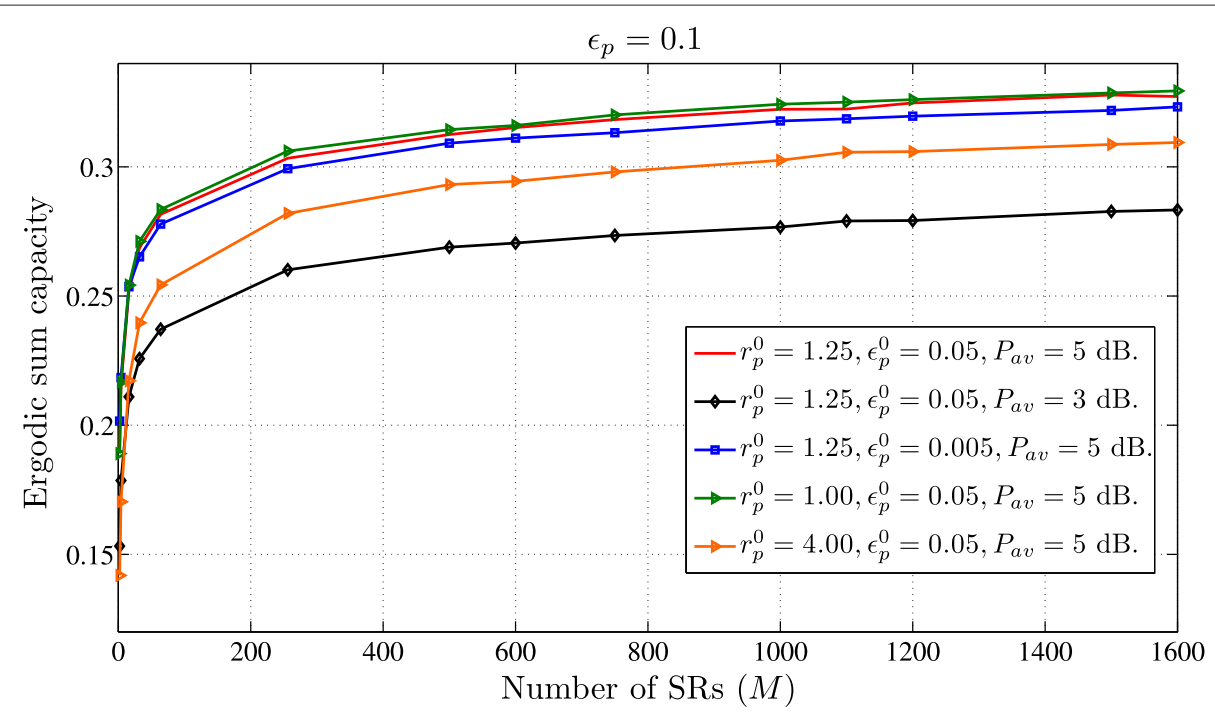

Figure 3 The effect of related parameters on SBS sum throughput against $M$ for TCI with STPC.

\section{Conclusions}

In this paper, we have investigated the information theoretic results of the ergodic sum capacity in fading cognitive broadcast channels, sharing the same frequency band as a delay sensitive PU. Under an outage probability constraint at the primary receiver, we have derived optimal power allocation strategies to maximize the SBS ergodic sum capacity, under an average (long-term) transmit power constraint or a peak (short-term) transmit power constraint. As the duality result is not directly applicable, we established a new approach to show that the opportunistic scheduling structure is also optimal under the primary outage probability constraint. This result allowed us to reformulate the ergodic sum capacity expression as a concave function, and the corresponding optimal power policies were derived by using a probabilistic power allocation technique. Under these opportunistic optimal power allocation schemes, we have also analyzed how the SBS sum throughput scales as the number of secondary receivers goes to infinity when all relevant channels undergo independent Rayleigh fading. These asymptotic capacity scaling laws are derived under the assumption that the PU uses a truncated channel inversion policy. Rigorous theoretical analyses show that the SBS sum throughput scales like $\epsilon_{p} \log (\log M)$. Further, an asymptotically optimal reduced feedback scheme is

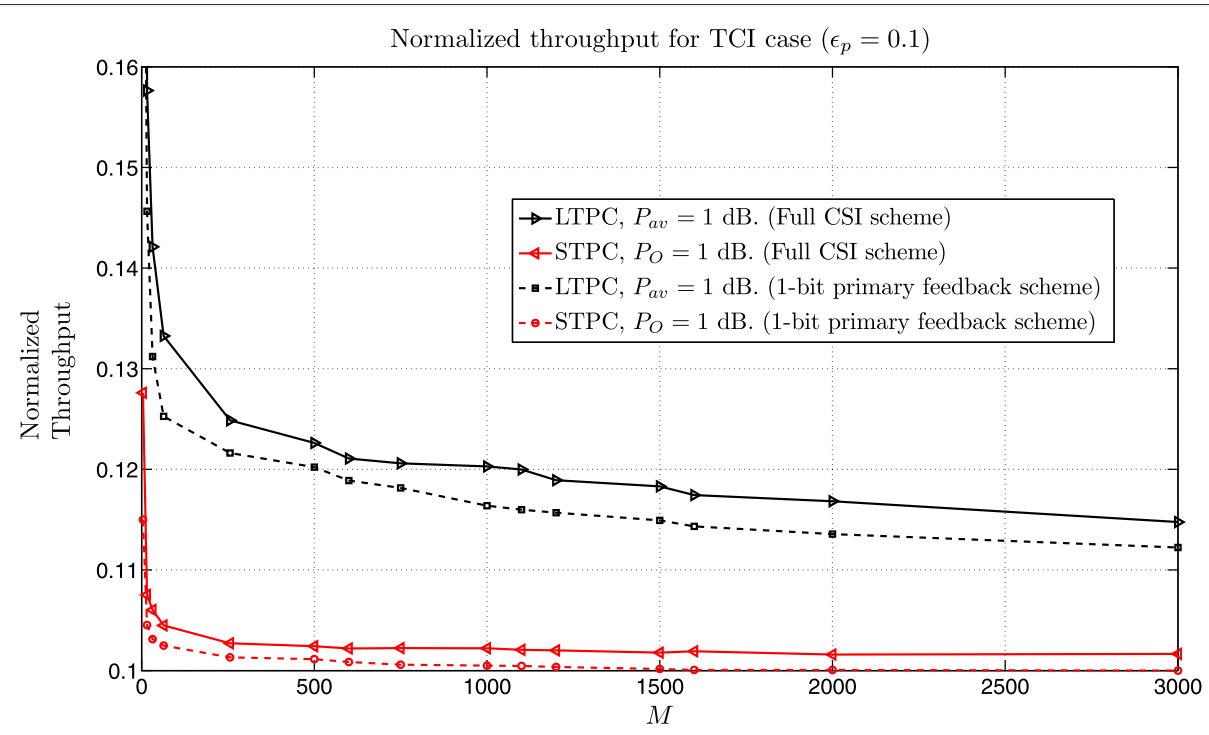

Figure 4 Normalized SBS sum throughput in TCI power policy at the PU. 
proposed. With only a 1-bit feedback from the primary receiver, we have shown that the SBS sum throughput for this suboptimal scheme retains like $\epsilon_{p} \log (\log M)$ scaling property of the optimal schemes.

\section{Appendix 1: opportunistic scheduling structure of the optimal power policy}

Prior to proving that the optimal power policy has an opportunistic scheduling structure, the following Lemma is required.

Lemma 1. For a non-negative vector $\mathbf{x}=\left[x_{1}, x_{2}\right.$, $\left.\ldots, x_{M}\right]^{T}$ and a non-negative vector $\gamma=$ $\left[\gamma_{1}, \gamma_{2}, \ldots, \gamma_{M}\right]^{T}$ where $\gamma_{1}<\gamma_{2}<\ldots<\gamma_{M}$, we have

$$
\sum_{i=1}^{M} \log \left(1+\frac{\gamma_{i} x_{i}}{1+\gamma_{i} \sum_{k=i+1}^{M} x_{k}}\right)<\log \left(1+\gamma_{M} \sum_{i=1}^{M} x_{i}\right)
$$

Proof. We will prove (21) by using induction. For $M=2$, $\log \left(1+\frac{\gamma_{1} x_{1}}{1+\gamma_{1} x_{2}}\right)+\log \left(1+\gamma_{2} x_{2}\right)=\log \left(1+\gamma_{1} \sum_{i=1}^{2} x_{i}\right)+$ $\log \left(\frac{1+\gamma_{2} x_{2}}{1+\gamma_{1} x_{2}}\right)$. Since $\log \left(\frac{1+\gamma_{2} x_{2}}{1+\gamma_{1} x_{2}}\right)$ is increasing in $x_{2}$ as $\gamma_{2}>\gamma_{1}$, we have $\log \left(\frac{1+\gamma_{2} x_{2}}{1+\gamma_{1} x_{2}}\right)<\log \left(\frac{1+\gamma_{2} \sum_{i=1}^{2} x_{i}}{1+\gamma_{1} \sum_{i=1}^{2} x_{i}}\right)$. Hence, we have $\log \left(1+\frac{\gamma_{1} x_{1}}{1+\gamma_{1} x_{2}}\right)+\log \left(1+\gamma_{2} x_{2}\right)<$ $\log \left(1+\gamma_{2} \sum_{i=1}^{2} x_{i}\right)$

Thus, (21) is true for $M=2$. Now suppose that (21) is true for $M=K$, we have to show that (21) is also true for $M=K+1$ :

$$
\begin{aligned}
& \sum_{i=1}^{K+1} \log \left(1+\frac{\gamma_{i} x_{i}}{1+\gamma_{i} \sum_{k=i+1}^{K+1} x_{k}}\right) \\
&=\left[\sum_{i=1}^{K} \log \left(1+\frac{\gamma_{i} x_{i}}{1+\gamma_{i} x_{K+1}+\gamma_{i} \sum_{k=i+1}^{K} x_{k}}\right)\right] \\
&+ {\left[\sum_{i=1}^{K} \log \left(1+\gamma_{K+1} x_{K+1}\right)\right.} \\
&+\log \left(1+\gamma_{K+1} x_{K+1}^{\prime}\right)
\end{aligned}
$$

where $\gamma_{i}^{\prime}=\frac{\gamma_{i}}{1+\gamma_{i} x_{K+1}}$. For a fixed $x_{K+1}, \gamma_{i}^{\prime}$ is an increasing function of $\gamma_{i}$, so we have $\gamma_{i+1}^{\prime}>\gamma_{i}^{\prime}$. Then, apply (21) for $M=K$, we obtain

$$
\begin{aligned}
& \sum_{i=1}^{K+1} \log \left(1+\frac{\gamma_{i} x_{i}}{1+\gamma_{i} \sum_{k=i+1}^{K+1} x_{k}}\right) \\
& \quad<\log \left(1+\gamma_{K}^{\prime} \sum_{i=1}^{K} x_{i}\right)+\log \left(1+\gamma_{K+1} x_{K+1}\right) \\
& =\log \left(1+\frac{\gamma_{i} \sum_{i=1}^{K} x_{i}}{1+\gamma_{i} x_{K+1}}\right)+\log \left(1+\gamma_{K+1} x_{K+1}\right) \\
& \quad=\log \left(1+\gamma_{K} \sum_{i=1}^{K+1} x_{i}\right)+\log \left(\frac{1+\gamma_{K+1} x_{K+1}}{1+\gamma_{K} x_{K+1}}\right) \\
& <\log \left(1+\gamma_{K+1} \sum_{i=1}^{K+1} x_{i}\right)
\end{aligned}
$$

Again, the last inequality is due to the fact that $\log \left(\frac{1+\gamma_{K+1} x_{K+1}}{1+\gamma_{K} x_{K+1}}\right)$ is increasing in $x_{K+1}$. Hence, it follows that (21) is also true for $M=K+1$, thus completing the proof.

Lemma 2. Let $\tilde{\mathbf{P}}(\chi)$ be the optimal power policy of the problem (5). Then, $\tilde{\mathbf{P}}(\boldsymbol{\chi})$ has an opportunistic scheduling structure; i.e. for each channel state $\chi$, the SBS allocates power only to the user with maximum $z_{i}$ and zero power to other remaining users.

Proof. We will prove Lemma 2 by using contradiction. Let $i^{*}=\arg \max z_{i}$. Suppose that the optimal power policy $\tilde{\mathbf{P}}(\chi)$ does not possess an opportunistic scheduling structure, i.e. the SBS allocates power to more than one users. Then, we construct another power policy with an opportunistic scheduling structure $\tilde{\mathbf{P}}^{\prime}(\boldsymbol{\chi})$ such that $\tilde{P}_{i^{*}}^{\prime}(\boldsymbol{\chi})=$ $\sum_{i=1}^{M} \tilde{P}_{i}(\boldsymbol{\chi})$ and $\tilde{P}_{i}^{\prime}(\boldsymbol{\chi})=0$ for $i \neq i^{*}$. It is worth noting that $\sum_{i=1}^{M} \tilde{P}_{\pi(i)}^{\prime}(\chi)=\sum_{i=1}^{M} \tilde{P}_{\pi(i)}(\chi)$. Therefore, the new power policy $\tilde{\mathbf{P}}^{\prime}(\boldsymbol{\chi})$ does not affect the power consumption at the SBS and the outage/non-outage status in the primary link. 
The instantaneous sum-rate from both power policies $\tilde{\mathbf{P}}(\boldsymbol{\chi})$ and $\tilde{\mathbf{P}}^{\prime}(\boldsymbol{\chi})$ are represented by $r_{s}^{\mathrm{BC}}(\boldsymbol{\chi}, \tilde{\mathbf{P}}(\boldsymbol{\chi}))$ and $r_{s}^{\mathrm{BC}}\left(\boldsymbol{\chi}, \tilde{\mathbf{P}}^{\prime}(\boldsymbol{\chi})\right)$. By using Lemma 1, we have

$$
\begin{aligned}
r_{s}^{\mathrm{BC}}(\boldsymbol{\chi}, \tilde{\mathbf{P}}(\boldsymbol{\chi})) & =\sum_{i=1}^{M} \log \left(1+\frac{z_{\pi(i)} \tilde{P}_{\pi(i)}(\chi)}{1+z_{\pi(i)} \sum_{k=i+1}^{M} \tilde{P}_{\pi(k)}(\boldsymbol{\chi})}\right) \\
& <\log \left(1+z_{\pi(M)} \sum_{i=1}^{M} \tilde{P}_{\pi(i)}^{\prime}(\boldsymbol{\chi})\right) \\
& =r_{s}^{\mathrm{BC}}\left(\boldsymbol{\chi}, \tilde{\mathbf{P}}^{\prime}(\boldsymbol{\chi})\right)
\end{aligned}
$$

which contradicts the optimality of $\tilde{\mathbf{P}}(\chi)$. Thus, it implies that the following opportunistic scheduling structure is optimal:

$$
P_{i}(\chi)= \begin{cases}\breve{P}(\chi), & i=\arg \max _{m \in \mathcal{I}} z_{m} \\ 0, & \text { otherwise }\end{cases}
$$

(25) suggests that the SBS allocates the transmit power, $\breve{P}(\chi)$, to the SR with the maximum $z_{m}$, where ties can be broken arbitrarily.

\section{Appendix 2: optimal solution of $\breve{P}^{*}(\chi)$ for LTPC}

By the opportunistic scheduling structure shown in Appendix 1, the original non-convex problem in (7) can be converted to convex problem as shown in (11). However, there is no closed-form expression of the primary outage probability function in POC. Hence, the convex optimization technique is not directly applicable. By using the same technique as in $[10,11]$, the following result in Lemma 3 is required.

Lemma 3. The optimal solution of the problem (11) can be expressed by $\breve{P}^{*}(\boldsymbol{\chi})=w(\boldsymbol{\chi}) \breve{p}_{1}(\boldsymbol{\chi})+(1-w(\boldsymbol{\chi})) \breve{p}_{2}(\boldsymbol{\chi})$, where $E[w(\chi)] \geq 1-\epsilon_{p}, E\left[\breve{P}^{*}(\boldsymbol{\chi})\right] \leq P_{a v}$ and $r_{p}\left(\boldsymbol{\chi}, \breve{p}_{1}(\boldsymbol{\chi})\right) \geq r_{p}^{0}$ for all $\chi$.

Proof. Following a similar procedure to that in [11], we will show that an optimal power control for (11) can be constructed by randomizing between two deterministic power allocation schemes, i.e. $\breve{p}_{1}(\boldsymbol{\chi})=E\left[\breve{P}(\boldsymbol{\chi}) \mid r_{p}(\boldsymbol{\chi}, \breve{p}(\boldsymbol{\chi})) \geq r_{p}^{0}\right]$ and $\breve{p}_{2}(\boldsymbol{\chi})=E\left[\breve{P}(\boldsymbol{\chi}) \mid r_{p}(\boldsymbol{\chi}, \breve{p}(\boldsymbol{\chi}))<r_{p}^{0}\right]$. The probability of using the power policy $\breve{p}_{1}(\chi)$ is indicated by the weighting function $w(\chi)$, which can be expressed as $w(\boldsymbol{\chi})=\operatorname{Pr}\left\{r_{p}(\boldsymbol{\chi}, \breve{P}(\boldsymbol{\chi})) \geq r_{p}^{0} \mid \boldsymbol{\chi}\right\}$ and therefore the probability of using the power policy $\breve{p}_{2}(\chi)$ equals $1-w(\boldsymbol{\chi})$.
We first show that given an arbitrary feasible probabilistic power scheme $\breve{P}(\chi)$, we can always construct another feasible scheme $\breve{P}^{\prime}(\chi)$ which is randomized among deterministic power schemes $\breve{p}_{k}(\chi), \quad k=1,2$ with a time-sharing factor $w(\chi)$ and performs equally well or better than $\breve{P}(\chi)$. Since $\breve{P}(\chi)$ is feasible, $\breve{P}(\chi)$ satisfies all the constraints, i.e. $E[\breve{P}(\boldsymbol{\chi})] \leq P_{\text {av }}$ and $\operatorname{Pr}\left\{\log \left(1+\frac{g P_{p}(g)}{N_{0}+\beta \breve{P}(\boldsymbol{\chi})}\right)<r_{p}^{0}\right\} \leq \epsilon_{p}$. Note that when $r_{p}(\chi, \breve{p}(\chi)) \geq r_{p}^{0}$, it implies that $\breve{p}(\chi) \leq \frac{1}{\beta}\left(\frac{g P_{p}(g)}{\exp \left(r_{p}^{0}\right)-1}-N_{0}\right)^{+}$i.e. the possible solution lies in a halfspace. Therefore, $\breve{p}_{1}(\chi)=$ $E\left[\breve{P}(\boldsymbol{\chi}) \mid r_{p}(\boldsymbol{\chi}, \breve{p}(\boldsymbol{\chi})) \geq r_{p}^{0}\right] \leq \frac{1}{\beta}\left(\frac{g P_{p}(g)}{\exp \left(r_{p}^{0}\right)-1}-N_{0}\right)^{+}$, i.e. a convex combination of the possible solutions in that halfspace weighted by the probability that each solution can happen also lies in that halfspace. So, $\log \left(1+\frac{g P_{p}(g)}{N_{0}+\beta \ddot{p}_{1}(\boldsymbol{x})}\right) \geq r_{p}^{0}$.

Thus, $\breve{P}^{\prime}(\chi)$ satisfies POC since $\operatorname{Pr}\left\{\breve{P}^{\prime}(\chi)=\breve{p}_{1}(\chi)\right.$

$\mid \chi\}=w(\chi)$ and then it can be shown that $E\left[\operatorname{Pr}\left\{\log \left(1+\frac{g P_{p}(g)}{N_{0}+\beta \check{P}^{\prime}(\boldsymbol{\chi})}\right) \geq r_{p}^{0} \mid \chi\right\}\right] \geq E[w(\boldsymbol{\chi})] \geq 1-\epsilon_{p}$.

Next, we can show that $\breve{P}^{\prime}(\chi)$ also satisfies LTPC since $E\left[\breve{P}^{\prime}(\boldsymbol{\chi})\right]=E\left[w(\boldsymbol{\chi}) \breve{p}_{1}(\boldsymbol{\chi})+(1-w(\boldsymbol{\chi})) \breve{p}_{2}(\boldsymbol{\chi})\right]=E[\breve{P}(\boldsymbol{\chi})] \leq$ $P_{\mathrm{av}}$.

Due to concavity of $r_{s}(\chi, \breve{P}(\chi))$ in (11), we can apply Jensen's inequality to arrive at the final result that $E\left[r_{s}\left(\boldsymbol{\chi}, \breve{P}^{\prime}(\boldsymbol{\chi})\right)\right] \geq E\left[r_{s}(\boldsymbol{\chi}, \breve{P}(\boldsymbol{\chi}))\right]$, i.e. $\breve{P}^{\prime}(\boldsymbol{\chi})$ achieves higher ergodic sum rate than $\breve{P}(\chi)$.

Reformulating (11) by using Lemma 3, we obtain the following optimization problem:

$$
\begin{aligned}
& \max _{\breve{p}_{k}(\boldsymbol{\chi}), w(\boldsymbol{\chi})} E\left[w(\boldsymbol{\chi}) r_{s}\left(\boldsymbol{\chi}, \breve{p}_{1}(\boldsymbol{\chi})\right)+(1-w(\boldsymbol{\chi})) r_{s}\left(\boldsymbol{\chi}, \breve{p}_{2}(\boldsymbol{\chi})\right)\right] \\
& \text { s.t. } E\left[w(\boldsymbol{\chi}) \breve{p}_{1}(\boldsymbol{\chi})+(1-w(\boldsymbol{\chi})) \breve{p}_{2}(\boldsymbol{\chi})\right] \leq P_{\mathrm{av}} \\
& E[w(\chi)] \geq 1-\epsilon_{p} \\
& w(\chi)\left[\mathscr{P}_{p}(g)-\beta \breve{p}_{1}(\chi)\right] \geq 0 \\
& \breve{p}_{k}(\chi) \succeq 0, \quad \forall k \in\{1,2\} \\
& 0 \leq w(\chi) \leq 1
\end{aligned}
$$

Using a similar variable transformation technique as in [10] and [11], the objective function can be proved to be concave while the other constraints are linear. Hence, the problem (26a) can be solved by the necessary and sufficient Karush-Kuhn-Tucker (KKT) optimality conditions. Applying the KKT conditions and the fact that channel state is continuous, the optimal 
solutions of $\breve{p}_{1}^{*}(\boldsymbol{\chi}), \breve{p}_{2}^{*}(\boldsymbol{\chi})$, and $w^{*}(\boldsymbol{\chi})$ are expressed as follows:

$$
\begin{aligned}
& \breve{p}_{1}^{*}(\chi)=\left\{\begin{array}{l}
p_{\mathrm{WF}}^{*}(\chi), w^{*}(\chi)=1, p_{\mathrm{WF}}^{*}(\chi) \leq p_{\mathrm{RP}}^{*}(\chi) \\
p_{\mathrm{RP}}^{*}(\chi), w^{*}(\chi)=1, p_{\mathrm{WF}}^{*}(\chi)>p_{\mathrm{RP}}^{*}(\chi)
\end{array}\right. \\
& \breve{p}_{2}^{*}(\chi)=p_{\mathrm{WF}}^{*}(\chi), \quad w^{*}(\chi)=0 \\
& w^{*}(\boldsymbol{\chi})=\left\{\begin{array}{l}
1, r_{s}\left(\boldsymbol{\chi}, \breve{p}_{1}^{*}(\boldsymbol{\chi})\right)-\Lambda^{*} \breve{p}_{1}^{*}(\boldsymbol{\chi})+S^{*}>r_{s}\left(\boldsymbol{\chi}, \breve{p}_{2}^{*}(\boldsymbol{\chi})\right)-\Lambda^{*} \breve{p}_{2}^{*}(\boldsymbol{\chi}) \\
0, r_{s}\left(\boldsymbol{\chi}, \breve{p}_{1}^{*}(\boldsymbol{\chi})\right)-\Lambda^{*} \breve{p}_{1}^{*}(\boldsymbol{\chi})+S^{*}<r_{s}\left(\boldsymbol{\chi}, \breve{p}_{2}^{*}(\boldsymbol{\chi})\right)-\Lambda^{*} \breve{p}_{2}^{*}(\boldsymbol{\chi})
\end{array}\right.
\end{aligned}
$$

where $p_{\mathrm{WF}}^{*}(\chi)=\left(\frac{1}{\Lambda^{*}}-\frac{1}{z_{\max }}\right)^{+}$. Finally, by applying the opportunistic scheduling structure shown in (25), we have the result in Theorem 1 .

\section{Appendix 3: optimal solution of $\breve{P}^{*, \text { st }}(\chi)$ for STPC}

Similar to Section 4.1, the objective function in (11) is concave over $\breve{P}^{\text {st }}(\chi)$. To apply the same technique as in Appendix 3, the following result in Lemma 4 is required.

Lemma 4. The optimal solution of the problem (16) can be expressed by $\breve{P}^{*, s t}(\chi)=w^{s t}(\chi) \breve{p}_{1}^{s t}(\chi)+(1-$ $\left.w^{s t}(\chi)\right) \breve{p}_{2}^{s t}(\chi)$, where $E\left[w^{s t}(\chi)\right] \geq 1-\epsilon_{p}, P^{*, s t}(\chi) \leq P_{O}$ and $r_{p}\left(\chi, \breve{p}_{1}^{s t}(\chi)\right) \geq r_{p}^{0}$ for all $\chi$.

Proof. Following a similar procedure to the proof of Lemma 3, we can prove that an optimal power control for (16) can be constructed by randomizing between two deterministic power allocation schemes, i.e. $\breve{p}_{1}^{\text {st }}(\boldsymbol{\chi})=E\left[\breve{P}^{\text {st }}(\boldsymbol{\chi}) \mid r_{p}\left(\boldsymbol{\chi}, \breve{p}^{\text {st }}(\boldsymbol{\chi})\right) \geq r_{p}^{0}\right]$ and $\breve{p}_{2}^{\text {st }}(\chi)=E\left[\breve{P}^{\text {st }}(\chi) \mid r_{p}\left(\chi, \breve{p}^{\text {st }}(\chi)\right)<r_{p}^{0}\right]$. The probability of using the power policy $\breve{p}_{1}^{\text {st }}(\chi)$ is indicated by the weighting function $w^{\text {st }}(\chi)$, which can be expressed as $w^{\text {st }}(\chi)=\operatorname{Pr}\left\{r_{p}\left(\chi, \breve{P}^{\text {st }}(\chi)\right) \geq r_{p}^{0} \mid \chi\right\}$, and therefore the probability of using the power policy $\breve{p}_{2}^{\text {st }}(\chi)$ equals $1-w^{\text {st }}(\boldsymbol{\chi})$.

By Lemma 4, we can get the reformulated version of (16). Following the same procedure as in the LTPC case in Appendix 2, we can apply the necessary and sufficient KKT conditions and the fact that the fading channel state is continuous, use the opportunistic scheduling structure in (25) and arrive at the optimal power policy summarized in Theorem 2.

\section{Appendix 4: proof of Theorem 3}

Here the channel state partitioning result from Table 1 will be used first to derive some properties related to the Lagrange multipliers $\Lambda^{*}$ and $S^{*}$ as the $M$ grows large. As discussed before, we assume that $S^{*}>0$. From Table 1, we can separate the regions representing fading channel sets 2 and 3 by setting $\mathscr{B}_{1, \chi}^{B}=$ $\mathscr{B}_{2, \boldsymbol{\chi}}^{B}$, where $\mathscr{B}_{1, \chi}^{B}=S^{*}, \mathscr{B}_{2, \chi}^{B}=\log \left(1+z_{\max } p_{\mathrm{WF}}^{*}\right)-$ $\Lambda^{*} p_{\mathrm{WF}}^{*}$, and $p_{\mathrm{WF}}^{*}=\left(\frac{1}{\Lambda^{*}}-\frac{1}{z_{\max }}\right)^{+}$. Thus, the boundary between channel sets given by cases 2 and 3 is given by

$S^{*}=\log \left(\frac{z_{\max }}{\Lambda^{*}}\right)-\left(1-\frac{\Lambda^{*}}{z_{\max }}\right)=-\log (\Omega)+\Omega-1=t(\Omega)$

where $\Omega=\frac{\Lambda^{*}}{z_{\max }}$ and $0 \leq \Omega \leq 1$. It is easy to show that $t(\Omega)$ is a non-increasing function in $\Omega$ when $0<\Omega \leq 1$. Thus, the channel sets for cases 1, 2, 3 in Table 1 can also be expressed as follows:

$$
\begin{aligned}
\tilde{\mathcal{S}}_{1} & =\left\{g<g_{T}\right\} \tilde{\mathcal{S}}_{2}=\left\{g \geq g_{T}, 0 \leq \Omega \leq t^{-1}\left(S^{*}\right)\right\} \\
& =\left\{g \geq g_{T}, \frac{\Lambda^{*}}{t^{-1}\left(S^{*}\right)} \leq z_{\max }<\infty\right\} \\
\tilde{\mathcal{S}}_{3} & =\left\{g \geq g_{T}, 0 \leq z_{\max } \leq \frac{\Lambda^{*}}{t^{-1}\left(S^{*}\right)}\right\}
\end{aligned}
$$

where $t^{-1}$ (.) represents the inverse function of $t$.

Lemma 5. $\Lambda^{*} \leq \frac{\epsilon_{p}}{P_{a v}}$ and $\Lambda^{*}$ is bounded away from 0 for all $M$.

Proof. First, we investigate the upper bound of $\Lambda^{*}$. As the average power constraint is always met, we have

$$
\begin{aligned}
P_{\mathrm{av}}= & \sum_{k=1}^{2} \operatorname{Pr}\left(\mathcal{S}_{k}\right) E\left[\left(\frac{1}{\Lambda^{*}}-\frac{1}{z_{\mathrm{max}}}\right)^{+} \mid \mathcal{S}_{k}\right] \\
& +\operatorname{Pr}\left(\mathcal{S}_{3}\right) E\left[p_{\mathrm{RP}}^{*}(\boldsymbol{\chi}) \mid \mathcal{S}_{3}\right] \\
\leq & \left(\frac{1}{\Lambda^{*}} \sum_{k=1}^{2} \operatorname{Pr}\left(\mathcal{S}_{k}\right)\right)+0=\frac{\epsilon_{p}}{\Lambda^{*}}
\end{aligned}
$$

Now suppose that $\lim _{M \rightarrow \infty} \Lambda_{M}^{*}=0$. Then, for an arbitrarily small $\epsilon>0$, there exists $M_{o}$ such that for $M \geq M_{o}$, $\Lambda_{M}^{*} \leq \epsilon$. Therefore, $\left(\frac{1}{\Lambda_{M}^{*}}-\frac{1}{z_{\max }}\right)^{+} \geq\left(\frac{1}{\epsilon}-\frac{1}{z_{\max }}\right)^{+}$. For an $M$ sufficiently large, $\frac{1}{z_{\max }}$ converges to 0 in probability, implying that $\left(\frac{1}{\epsilon}-\frac{1}{z_{\max }}\right)^{+}$converges to $\frac{1}{\epsilon}$ in probability. Hence, $\left(\frac{1}{\Lambda_{M}^{*}}-\frac{1}{z_{\max }}\right)^{+} \geq \frac{1}{\epsilon}$ with high probability. So, with an arbitrary small $\epsilon$, SU will violate the power constraint with high probability if $\lim _{M \rightarrow \infty} \Lambda_{M}^{*}=0$. Consequently, $\lim _{M \rightarrow \infty} \Lambda_{M}^{*}>0$. 
Next, we will show that when $M$ is large enough, $\Lambda_{M}^{*}$ is lower-bounded by $\frac{\epsilon_{p}^{0}}{P_{\mathrm{av}}}$.

$$
\begin{aligned}
P_{\mathrm{av}} & =\sum_{k=1}^{2} \operatorname{Pr}\left(\mathcal{S}_{k}\right) E\left[\left(\frac{1}{\Lambda_{M}^{*}}-\frac{1}{z_{\max }}\right)^{+} \mid \mathcal{S}_{k}\right] \\
& >\operatorname{Pr}\left(\mathcal{S}_{1}\right) E\left[\left(\frac{1}{\Lambda_{M}^{*}}-\frac{1}{z_{\max }}\right)^{+} \mid \mathcal{S}_{1}\right] \\
& =\epsilon_{p}^{0} E\left[\left(\frac{1}{\Lambda_{M}^{*}}-\frac{1}{z_{\max }}\right)^{+} \mid \mathcal{S}_{1}\right]
\end{aligned}
$$

Also,

$$
\begin{aligned}
& \lim _{M \rightarrow \infty} E\left[\left(\frac{1}{\Lambda_{M}^{*}}-\frac{1}{z_{\max }}\right)^{+} \mid \mathcal{S}_{1}\right] \\
& =\lim _{M \rightarrow \infty}\left[\frac{1}{\Lambda_{M}^{*}}\left(1-F_{Z}^{M}\left(\Lambda_{M}^{*} \mid \mathcal{S}_{1}\right)\right)-\int_{z_{\max } \Lambda_{M}^{*}}^{\infty} \frac{1}{z_{\max }} d F_{Z}^{M}\left(z_{\max } \mid \mathcal{S}_{1}\right)\right] \\
& =\frac{1}{\Lambda_{M}^{*}}
\end{aligned}
$$

Note that the last equality from (33) is because of $\int_{z_{\max }=\Lambda_{M}^{*}}^{\infty} \frac{1}{z_{\max }} d F_{Z}^{M}\left(z_{\max } \mid \mathcal{S}_{1}\right)$ and $F_{Z}^{M}\left(\Lambda_{M}^{*} \mid \mathcal{S}_{1}\right)$ approach
zero as $M \rightarrow \infty$.

Lemma 6. For $\epsilon_{p}>\epsilon_{p}^{0}, \frac{1}{t^{-1}\left(S^{*}\right)}=\Theta(\log M)$.

Proof. First, notice that the SBS will allocate transmission power only to the user with $z_{\max }$ and $\max \frac{h_{i}}{\frac{k_{1}}{g_{T}} \alpha_{i}+N_{0}} \leq$ $z_{\max } \leq \max \frac{h_{i}}{N_{0}}$ regardless the value of $g$. This fact allows us to study how the upper and lower bounds of $z_{\max }$ scales as $M$ grows large. By using the results from [22,37], we can show that $z_{\max }$ is upper-and-lower-bounded by $\log M$ as $M \rightarrow \infty$. Hence, $z_{\max }$ also scales as $\log M$ when $M$ tends to infinity.

Now we assume that $\frac{1}{t^{-1}\left(S^{*}\right)}$ grows faster than $\log M$ as $M \rightarrow \infty$. This implies that for a large enough $M$, $\operatorname{Pr}\left(\tilde{\mathcal{S}}_{2}\right)$ converges to 0 , which contradicts the fact that $\operatorname{Pr}\left(\tilde{\mathcal{S}}_{2}\right)=\epsilon_{p}-\epsilon_{p}^{0}>0$ for all $M$. Next, assume that $\frac{1}{t^{-1}\left(S^{*}\right)}$ grows slower than $\log M$. This implies that when $M$ is large enough, $\operatorname{Pr}\left(\tilde{\mathcal{S}}_{3}\right)$ converges to 0 , which contradicts the fact that $\operatorname{Pr}\left(\tilde{\mathcal{S}}_{3}\right)=1-\epsilon_{p}>0$ for all $M$. Thus, $\frac{1}{t^{-1}\left(S^{*}\right)}=\Theta(\log M)$.
Observe that the SBS gains no throughput from the region 3 . We will show that the asymptotic throughput achieved by the secondary network scales as $\epsilon_{p} \log (\log M)$, where the optimal power control policy in the region 1 contributes $\epsilon_{p}^{0} \log (\log M)$ of the optimal throughput scaling, while the remaining $\left(\epsilon_{p}-\epsilon_{p}^{0}\right) \log (\log M)$ is obtained from the optimal power control policy in the region 2 . In order to do so, the results derived above will facilitate us to arrive at the result in Theorem 3 for both (1) when $\epsilon_{p}=\epsilon_{p}^{0}$ (additional outage probability is not allowed) and (2) when $\epsilon_{p}>\epsilon_{p}^{0}$ (the delay-sensitive PU's link can tolerate an additional outage).

\section{When $\epsilon_{p}=\epsilon_{p}^{0}$}

In this case, region 2 shrinks to an empty set, i.e. $\operatorname{Pr}\left(\tilde{\mathcal{S}}_{2}\right)=$ $\epsilon_{p}-\epsilon_{p}^{0}=0$, and the overall optimal throughput scaling is due to transmission power in region 1.

Clearly, as $M$ becomes large, the optimal throughput scaling is due to the effect of the scaling result of $z_{\max }=h_{\max }$ with the number of SRs in $\tilde{\mathcal{S}}_{1}$. Moreover, we know that $\Lambda^{*} \leq \frac{\epsilon_{p}}{P_{\mathrm{av}}}$ and $\Lambda^{*}$ is bounded away from 0 for all $M$ (see Lemma 5.) Then, the SBS ergodic sum capacity under optimal power allocation is expressed as $C_{s}^{B C *}=E\left[\log \left(\frac{z_{\max }}{\Lambda^{*}}\right) 1_{\left\{z_{\max } \geq \Lambda^{*}, \tilde{\mathcal{S}}_{1}\right\}}\right]=\epsilon_{p}^{0} E\left[\log \left(\frac{h_{\max }}{\Lambda^{*}}\right)\right]=$ $\epsilon_{p} E\left[\log \left(\frac{h_{\max }}{\Lambda^{*}}\right)\right]$. As the maximum of $M$ independent and identically distributed exponentially distributed random variables, $\frac{h_{\max }}{\log M}$ converges in probability to 1 . (see Example 10.5.1 of [37]). Using this result and the rigorous techniques used in [22] to show convergence in mean from convergence in probability, we can conclude that $\lim _{M \rightarrow \infty} \frac{C_{s}^{B C *}}{\log (\log M)}=\epsilon_{p}$.

\section{When $\epsilon_{p}>\epsilon_{p}^{0}$}

Note that the throughput $C_{s}^{\mathrm{BC}}$ can be written as

$$
\begin{aligned}
C_{s}^{\mathrm{BC}}= & E\left[\log \frac{z_{\max }}{\Lambda^{*}} 1_{\left\{g<g_{T}, z_{\max } \geq \Lambda^{*}\right\}}\right] \\
& +E\left[\log \frac{z_{\max }}{\Lambda^{*}} 1_{\left\{g \geq g_{T}, z_{\max } \geq \frac{\Lambda^{*}}{t^{-1}\left(S^{*}\right)}\right\}}\right]
\end{aligned}
$$

Note that in the previous subsection, we showed that $E\left[\log \frac{z_{\max }}{\Lambda^{*}} 1_{\left\{g<g_{T}, z_{\max } \geq \Lambda^{*}\right\}}\right]$ scales according to $\epsilon_{p}^{0} \log (\log M)$ when $M \rightarrow \infty$. Now, we show that $E\left[\log \frac{z_{\max }}{\Lambda^{*}} 1_{\left\{g \geq g_{T}, z_{\max } \geq \frac{\Lambda^{*}}{t^{-1}\left(S^{*}\right)}\right\}}\right]$ scales according to $\left(\epsilon_{p}-\right.$ $\left.\epsilon_{p}^{0}\right) \log (\log M)$. 
Upper bound An upper bound on $E\left[\log \frac{z_{\max }}{\Lambda^{*}}\right.$ $\left.1_{\left\{g \geq g_{T}, z_{\max } \geq \frac{\Lambda^{*}}{t^{-1}\left(S^{*}\right)}\right\}}\right]$ can be obtained as shown in (36).

$$
\begin{aligned}
& E\left[\log \frac{z_{\max }}{\Lambda^{*}} 1_{\left\{g \geq g_{T}, z_{\max } \geq \frac{\Lambda^{*}}{t^{-1}\left(S^{*}\right)}\right\}}\right] \\
& =E\left[\log \frac{z_{\max }}{\Lambda^{*}} 1_{\left\{z_{\max } \leq \log M, g \geq g_{T}, z_{\max } \geq \frac{\Lambda^{*}}{t^{-1}\left(S^{*}\right)}\right\}}\right]+\sum_{i=1}^{\infty} \\
& \times E\left[\log \frac{z_{\max }}{\Lambda^{*}} 1_{\left\{i \log M \leq z_{\max } \leq(i+1) \log M, g \geq g_{T}, z_{\max } \geq \frac{\Lambda^{*}}{t^{-1}\left(S^{*}\right)}\right\}}\right] \\
& \leq \log \left(\frac{\log M}{\Lambda^{*}}\right) \operatorname{Pr}\left(z_{\max } \leq \log M, g \geq g_{T}, z_{\max } \geq \frac{\Lambda^{*}}{t^{-1}\left(S^{*}\right)}\right) \\
& +\sum_{i=1}^{\infty} \log \left(\frac{(i+1) \log M}{\Lambda^{*}}\right) \\
& \times \operatorname{Pr}\left(z_{\max } \geq i \log M, g \geq g_{T}, z_{\max } \geq \frac{\Lambda^{*}}{t^{-1}\left(S^{*}\right)}\right) \\
& \stackrel{(a)}{\leq} \log \left(\frac{\log M}{\Lambda^{*}}\right) \operatorname{Pr}\left(g \geq g_{T}, z_{\max } \geq \frac{\Lambda^{*}}{t^{-1}\left(S^{*}\right)}\right) \\
& +\sum_{i=1}^{\infty} \log \left(\frac{(i+1) \log M}{\Lambda^{*}}\right) \operatorname{Pr}\left(z_{\max } \geq i \log M\right) \\
& \stackrel{(b)}{\leq}\left(\epsilon_{p}-\epsilon_{p}^{0}\right) \log \left(\frac{\log M}{\Lambda^{*}}\right)+\sum_{i=1}^{\infty} \log \left(\frac{(i+1) \log M}{\Lambda^{*}}\right) O\left(\frac{e^{O(i)}}{M^{i} O(i)}\right)
\end{aligned}
$$

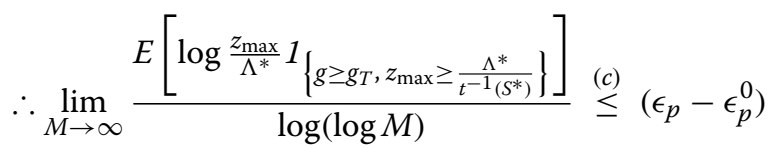

The inequality $(a)$ is from the fact that

$$
\begin{aligned}
& \operatorname{Pr}\left(z_{\max } \leq \log M, g \geq g_{T}, z_{\max } \geq \frac{\Lambda^{*}}{t^{-1}\left(S^{*}\right)}\right) \\
& \quad \leq \operatorname{Pr}\left(g \geq g_{T}, z_{\max } \geq \frac{\Lambda^{*}}{t^{-1}\left(S^{*}\right)}\right), \\
& \operatorname{Pr}\left(z_{\max } \geq i \log M, g \geq g_{T}, z_{\max } \geq \frac{\Lambda^{*}}{t^{-1}\left(S^{*}\right)}\right) \\
& \quad \leq \operatorname{Pr}\left(z_{\max } \geq i \log M\right) .
\end{aligned}
$$

For $(b)$, we use the result in [38] (see proof of Theorem 1 in [38]), i.e. $\operatorname{Pr}\left(z_{\max } \geq i \log M\right) \leq O\left(\frac{e^{O(i)}}{M^{i} O(i)}\right)$ because $z_{\max }$ grows as $\log M$ when $M \rightarrow \infty$. In [38], it is also shown that $\sum_{i=1}^{\infty} \log \left(\frac{(i+1) \log M}{\Lambda^{*}}\right) O\left(\frac{e^{O(i)}}{M^{i} O(i)}\right)$ is finite, leading to the result in $(c)$. Thus, we have

$$
\begin{aligned}
\lim _{M \rightarrow \infty} \frac{C_{s}^{\mathrm{BC}}}{\log (\log M)}= & \lim _{M \rightarrow \infty} \frac{E\left[\log \frac{z_{\max }}{\Lambda^{*}} 1_{\left\{g \geq g_{T}, z_{\max } \geq \Lambda^{*}\right\}}\right]}{\log (\log M)} \\
& +\lim _{M \rightarrow \infty} \frac{E\left[\log \frac{z_{\max }}{\Lambda^{*}} 1_{\left.\left\{\geq g_{T}, z_{\max } \geq \frac{\Lambda^{*}}{t^{-1}\left(S^{*}\right)}\right\}\right]}\right.}{\log (\log M)} \\
\leq & \epsilon_{p}^{0}+\left(\epsilon_{p}-\epsilon_{p}^{0}\right) \\
= & \epsilon_{p}
\end{aligned}
$$

Lower bound We use (38) to find a lower bound on $E\left[\log \frac{z_{\max }}{\Lambda^{*}} 1_{\left\{g \geq g_{T}, z_{\max } \geq \frac{\Lambda^{*}}{t^{-1}\left(S^{*}\right)}\right\}}\right]$ as follows:

$$
\begin{aligned}
& E\left[\log \frac{z_{\max }}{\Lambda^{*}} 1_{\left\{g \geq g_{T}, z_{\max } \geq \frac{\Lambda^{*}}{t^{-1}\left(S^{*}\right)}\right\}}\right] \\
& \quad \geq E\left[\log \left(\frac{1}{t^{-1}\left(S^{*}\right)}\right) 1_{\left\{g \geq g_{T}, z_{\max } \geq \frac{\Lambda^{*}}{t^{-1}\left(S^{*}\right)}\right\}}\right] \\
& \quad=\log \left(\frac{1}{t^{-1}\left(S^{*}\right)}\right) \operatorname{Pr}\left(\tilde{\mathcal{S}}_{2}\right) \\
& \quad=\left(\epsilon_{p}-\epsilon_{p}^{0}\right) \log \left(\frac{1}{t^{-1}\left(S^{*}\right)}\right)
\end{aligned}
$$

Then, we have

$$
\begin{aligned}
\lim _{M \rightarrow \infty} \frac{C_{s}^{\mathrm{BC}}}{\log (\log M)}= & \lim _{M \rightarrow \infty} \frac{E\left[\log \frac{z_{\max }}{\Lambda^{*}} 1_{\left.\left\{g<g_{T}, z_{\max } \geq \Lambda^{*}\right\}\right]}\right.}{\log (\log M)} \\
& +\lim _{M \rightarrow \infty} \frac{E\left[\log \frac{z_{\max }}{\Lambda^{*}} 1_{\left.\left\{g \geq g_{T}, z \max \geq \frac{\Lambda^{*}}{t^{-1}\left(S^{*}\right)}\right\}\right]}\right.}{\log (\log M)} \\
& =\epsilon_{p}^{0}+\lim _{M \rightarrow \infty} \frac{E\left[\log \frac{z_{\max }}{\Lambda^{*}} 1_{\left.\left\{g \geq g_{T}, z_{\max } \geq \frac{\Lambda^{*}}{t^{-1}\left(S^{*}\right)}\right\}\right]}\right.}{\log (\log M)} \\
& \geq \epsilon_{p}^{0}+\left(\epsilon_{p}-\epsilon_{p}^{0}\right)_{M \rightarrow \infty} \frac{\log \left(\frac{1}{t^{-1}\left(S^{*}\right)}\right)}{\log (\log M)} \\
& =\epsilon_{p}
\end{aligned}
$$

Note that the last equality in (39) follows from Lemma 6. Thus, it follows that $\lim _{M \rightarrow \infty} \frac{C_{s}^{\mathrm{BC}}}{\log (\log M)} \geq \epsilon_{p}$.

Combining the upper and lower bounds, we have the desired asymptotic throughput scaling result that $\lim _{M \rightarrow \infty} \frac{C_{s}^{\mathrm{BC}}}{\log (\log M)}=\epsilon_{p}$.

\section{Appendix 5: proof of Theorem 4}

As discussed before, we assume that $S^{*, s t}>0$. From Table 2, we find the boundary between regions 2 and 
3 by setting $\mathscr{B}_{1, \chi}^{B, \mathrm{st}}=\mathscr{B}_{2, \chi}^{B, \mathrm{st}}$, where $\mathscr{B}_{1, \chi}^{z B, \mathrm{st}}=S^{* \text {,st }}$ and $\mathscr{B}_{2, \chi}^{B, \text { st }}=\log \left(1+z_{\max } P_{O}\right)-\Lambda^{*, \text { st }}(\chi) P_{O}$. Note that for STPC, $\Lambda^{*, \mathrm{st}}(\chi)=\frac{z_{\max }}{1+z_{\max } P_{O}}$ if the power policy $P_{O}$ is applied. This results in the following expression for the boundary between regions corresponding to the fading channel states representing regions 2 and 3 :

$$
\begin{aligned}
S^{*, \text {st }} & =\log \left(1+z_{\max } P_{O}\right)-\frac{z_{\max } P_{O}}{1+z_{\max } P_{O}}=-\log (\omega)+\omega-1 \\
& =t(\omega)
\end{aligned}
$$

where $\omega=\frac{1}{1+z_{\max } P_{O}}$ and $0 \leq \omega \leq 1$. Further note that $t(\omega)$ is a non-increasing function in $\omega$ for $0<\omega \leq 1$. Thus, the regions corresponding to the various sets of the channel fading state in Table 2 can also be expressed as follows:

$$
\begin{aligned}
\tilde{\mathcal{S}}_{1} & =\left\{g<g_{T}\right\} \tilde{\mathcal{S}}_{2}=\left\{g \geq g_{T}, 0 \leq \omega \leq t^{-1}\left(S^{*, \mathrm{st}}\right)\right\} \\
& =\left\{g \geq g_{T}, \frac{1}{P_{O}}\left(\frac{1}{t^{-1}\left(S^{*, \mathrm{st}}\right)}-1\right) \leq z_{\max }<\infty\right\} \\
\tilde{\mathcal{S}}_{3} & =\left\{g \geq g_{T}, 0 \leq z_{\max } \leq \frac{1}{P_{O}}\left(\frac{1}{t^{-1}\left(S^{*, \mathrm{st}}\right)}-1\right)\right\} .
\end{aligned}
$$

For STPC, the optimal throughput scaling is also contributed by regions 1 and 2 because transmission in secondary network is halted in region 3 . In what follows, we show that $\epsilon_{p}^{0}$ fraction of the total optimal throughput scaling is from the optimal power policy in region 1, whereas the remaining $\left(\epsilon_{p}-\epsilon_{p}^{0}\right)$ fraction is due to data transmission in region 2 . We also use the result that $z_{\max }$ scales like $\log M$ as $M$ tends to infinity. The rest of the proof is divided into two parts: (i) $\epsilon_{p}=\epsilon_{p}^{0}$ and (ii) $\epsilon_{p}>\epsilon_{p}^{0}$.

\section{When $\epsilon_{p}=\epsilon_{p}^{0}$}

In this case, we have $\operatorname{Pr}\left(\tilde{\mathcal{S}}_{2}\right)=\epsilon_{p}-\epsilon_{p}^{0}=0$ and $z_{\max }=h_{\max }$ in $\tilde{\mathcal{S}}_{1}$. Then, the throughput from the SBS is expressed as $C_{s}^{\mathrm{BC} *}=E\left[\log \left(1+z_{\max } P_{O}\right) 1_{\left\{\tilde{\mathcal{S}}_{1}\right\}}\right]=$ $\epsilon_{p}^{0} E\left[\log \left(1+z_{\max } P_{O}\right)\right]$. By using the fact that $z_{\max }$ scales as $\log M$ as $M$ becomes large, we can conclude that $\lim _{M \rightarrow \infty} \frac{C_{s}^{\mathrm{BC} *}}{\log (\log M)}=\epsilon_{p}$.

\section{When $\epsilon_{p}>\epsilon_{p}^{0}$}

In this case, we also require the property that $t^{-1}\left(S^{*}\right)$ scales as $\log M$ when $M$ grows large (see Lemma 6). By using the fact that both $z_{\max }$ and $t^{-1}\left(S^{*}\right)$ scale as $\log M$, we can show that the upper and lower bounds of the optimal throughput in the secondary downlink network scale like $\epsilon_{p} \log (\log M)$. Note that the SBS ESC with optimal power allocation $C_{s}^{\mathrm{BC} *}$ can be expressed as

$$
\begin{aligned}
C_{s}^{\mathrm{BC} *}= & E\left[\log \left(1+z_{\max } P_{O}\right) 1_{\left\{g<g_{T}\right\}}\right] \\
& +E\left[\log \left(1+z_{\max } P_{O}\right) 1_{\left\{g \geq g_{T}, 1+z_{\max } P_{O} \geq \frac{1}{t^{-1}\left(S^{*}\right)}\right\}}\right]
\end{aligned}
$$

As shown previously, $E\left[\log \left(1+z_{\max } P_{O}\right) 1_{\left\{g<g_{T}\right\}}\right]$ scales as $\epsilon_{p}^{0} \log (\log M)$. Now, we will show that $E\left[\log \left(1+z_{\max } P_{O}\right) 1_{\left\{g \geq g_{T}, 1+z_{\max } P_{O} \geq \frac{1}{t^{-1}\left(S^{*}\right)}\right\}}\right]$ scales as $\left(\epsilon_{p}-\epsilon_{p}^{0}\right) \log (\log M)$.

Upper bound An upper bound on $E\left[\log \left(1+z_{\max } P_{O}\right)\right.$. $\left.1_{\left\{g \geq g_{T}, 1+z_{\max } P_{O} \geq \frac{1}{t^{-1}\left(S^{*}\right)}\right\}}\right]$ is shown in (43):

$$
\begin{aligned}
& E\left[\log \left(1+z_{\max } P_{O}\right) 1_{\left\{g \geq g_{T}, 1+z_{\max } P_{O} \geq \frac{1}{t^{-1}\left(S^{*}\right)}\right\}}\right] \\
& =E\left[\log \left(1+z_{\max } P_{O}\right) 1_{\left\{1 \leq z_{\max } \leq \log M, g \geq g_{T}, 1+z_{\max } P_{O} \geq \frac{1}{t^{-1}\left(S^{*}\right)}\right\}}\right]+\sum_{i=1}^{\infty} \\
& \times E\left[\log \left(1+z_{\max } P_{O}\right) 1_{\left\{i \log M \leq z_{\max } \leq(i+1) \log M, g \geq g_{T}, 1+z_{\max } P_{O} \geq \frac{1}{t^{-1}\left(S^{*}\right)}\right\}}\right] \\
& \leq \log \left(1+P_{O} \log M\right) \operatorname{Pr}\left(1 \leq z_{\max } \leq \log M, g \geq g_{T}, 1+z_{\max } P_{O} \geq \frac{1}{t^{-1}\left(S^{*}\right)}\right) \\
& +\sum_{i=1}^{\infty} \log \left(1+P_{O}(i+1) \log M\right) \\
& \times \operatorname{Pr}\left(i \log M \leq z_{\max } \leq(i+1) \log M, g \geq g_{T}, 1+z_{\max } P_{O} \geq \frac{1}{t^{-1}\left(S^{*}\right)}\right) \\
& \leq \log \left(1+P_{O} \log M\right) \operatorname{Pr}\left(g \geq g_{T}, 1+z_{\max } P_{O} \geq \frac{1}{t^{-1}\left(S^{*}\right)}\right) \\
& +\sum_{i=1}^{\infty} \log \left(1+P_{O}(i+1) \log M\right) \operatorname{Pr}\left(i \log M \leq z_{\max } \leq(i+1) \log M\right) \\
& \stackrel{(a)}{\leq}\left(\epsilon_{p}-\epsilon_{p}^{0}\right) \log \left(1+P_{O} \log M\right)+\sum_{i=1}^{\infty} \log \left(1+P_{O}(i+1) \log M\right) O\left(\frac{e^{O(i)}}{M^{i} O(i)}\right) \\
& \therefore \lim _{M \rightarrow \infty} \frac{E\left[\log \left(1+z_{\max } P_{O}\right) 1_{\left.\left\{g \geq g_{T}, 1+z_{\max } P_{O} \geq \frac{1}{t^{-1}\left(S^{*}\right)}\right\}\right]}\right.}{\log (\log M)} \stackrel{(b)}{\leq}\left(\epsilon_{p}-\epsilon_{p}^{0}\right)
\end{aligned}
$$

In (43), (a) follows the from the fact that $\operatorname{Pr}\left(z_{\max } \geq\right.$ $i \log M) \leq O\left(\frac{e^{O(i)}}{M^{i} O(i)}\right)$ because $z_{\max }$ grows like $\log \bar{M}$, while $(b)$ is from the fact that $\sum_{i=1}^{\infty} \log \left(1+P_{O}(i+1)\right.$ $\log M) O\left(\frac{e^{O(i)}}{M^{i} O(i)}\right)$ is finite [38].

Lower bound A lower bound can be computed in a similar fashion to (38) to yield 


$$
\begin{aligned}
& E\left[\log \left(1+z_{\max } P_{O}\right) 1_{\left\{g \geq g_{T}, 1+z_{\max } P_{O} \geq \frac{1}{t^{-1}\left(S^{*}\right)}\right\}}\right] \\
& \quad \geq\left(\epsilon_{p}-\epsilon_{p}^{0}\right) \log \left(\frac{1}{t^{-1}\left(S^{*}\right)}\right)
\end{aligned}
$$

We then apply Lemma 6, the results from (43) and (44), and the fact that $E\left[\log \left(1+z_{\max } P_{O}\right) 1_{\left\{g<g_{T}\right\}}\right]$ scales as $\epsilon_{p}^{0} \log (\log M)$ to show that $\lim _{M \rightarrow \infty} \frac{C_{s}^{\mathrm{BC} *}}{\log (\log M)}$ is upperbounded and lower-bounded by $\epsilon_{p}$, i.e. $\lim _{M \rightarrow \infty} \frac{C_{s}^{\mathrm{BC} *}}{\log (\log M)}=$ $\epsilon_{p}$.

\section{Competing interests}

The authors declare that they have no competing interests.

\section{Author details}

${ }^{1}$ Information Systems Technology and Design Pillar, Singapore University of Technology and Design, 20 Dover Drive, Singapore 138682, Singapore. ${ }^{2}$ Department of Engineering Science, Uppsala University, P.O. Box 534 Uppsala 751 21, Sweden. ${ }^{3}$ Department of Electrical and Electronic Engineering, The University of Melbourne, Parkville, Victoria 3010, Australia.

\section{Received: 10 October 2013 Accepted: 3 March 2014}

\section{Published: 6 March 2014}

\section{References}

1. Federal Communications Commission (FCC), Spectrum policy task force. Technical report, Federal Communications Commission (FCC). Rep. ET Docket no. 02-135, Nov 2002

2. J Mitola, Cognitive radio: an integrated agent architecture for software defined radio. PhD thesis (KTH, Stockholm, Sweden, 2000)

3. A Goldsmith, SA Jafar, I Marić, S Srinivasa, Breaking spectrum gridlock with cognitive radios: an information theoretic perspective. Proc. IEEE. 97(5), 894-914 (2009)

4. L Zhang, Y-C Liang, S Cui, Dynamic resource allocation in cognitive radio networks. IEEE Signal Process. Mag. 27(3), 102-114 (2010)

5. L Li, A Goldsmith, Capacity and optimal resource allocation for fading broadcast channels - part I: ergodic capacity. IEEE Trans. Inf. Theory. 47(3), 1083-1101 (2001)

6. L Li, A Goldsmith, Capacity and optimal resource allocation for fading broadcast channels - part II: outage capacity. IEEE Trans. Inf. Theory. 47(3), 1103-1127 (2001)

7. DNC Tse, Optimal power allocation over parallel Gaussian broadcast channels, in IEEE International Symposium on Information Theory (ISIT) (Ulm, July 1997)

8. N Jindal, S Vishwanath, A Goldsmith, On the duality of Gaussian multiple-access and broadcast channels. IEEE Trans. Inf. Theory. 50(5), 768-783 (2004)

9. R Zhang, S Cui, Y-C Liang, On ergodic sum capacity of fading cognitive multiple-access and broadcast channels. IEEE Trans. Inf. Theory. 55(11), 5161-5178 (2009)

10. J Luo, R Yates, P Spasojevic, Service outage based power rate allocation for parallel fading channels. IEEE Trans. Inf. Theory. 51(7), 2594-2611 (2005)

11. A Limmanee, S Dey, JS Evans, Service-outage capacity maximization in cognitive radio for parallel fading channels. IEEE Trans. Commun. 61(2), 507-520 (2013)

12. G Caire, G Taricco, E Biglieri, Optimum power control over fading channels. IEEE Trans. Inf. Theory. 45(5), 1468-1489 (1999)

13. A Limmanee, S Dey, Optimal power policy and throughput analysis in cognitive broadcast networks under primary's outage constraint, in The 8th International Workshop on Resource Allocation and Cooperation in Wireless Networks (RAWNET2012) (Paderborn, Germany, May 2012), pp. $391-397$

14. X Kang, R Zhang, Y-C Liang, HK Garg, Optimal power allocation strategies for fading cognitive radio channels with primary user outage constraint. IEEE J. Selected Areas Commun. 29(2), 374-383 (2011)
15. M Sharif, B Hassibi, On the capacity of MIMO broadcast channels with partial side information. IEEE Trans. Inf. Theory. 51(2), 506-522 (2005)

16. TW Ban, W Choi, BC Jung, DK Sung, Multi-user diversity in a spectrum sharing system. IEEE Trans. Wireless Commun. 8(1), 102-106 (2009)

17. H Wang, J Lee, S Kim, D Hong, Capacity of secondary users exploiting multispectrum and multiuser diversity in spectrum-sharing environments. IEEE Trans. Vehicular Technol. 59(2), 1030-1036 (2010)

18. R Zhang, Y-C Liang, Investigation on multiuser diversity in spectrum sharing based cognitive radio networks. IEEE Commun. Lett. 14(2), 133-135 (2010)

19. Y Li, A Nosratinia, Hybrid opportunistic scheduling in cognitive radio networks. IEEE Trans. Wireless Commun. 11(1), 328-337 (2012)

20. Y Li, A Nosratinia, Capacity limits of multiuser multiantenna cognitive networks. IEEE Trans. Inf. Theory. 58(7), 4493-4508 (2012)

21. K Huang, RW Heath, JG Andrews, Uplink SDMA with limited feedback: throughput scaling. EURASIP J. Adv. Signal Process. 2008, 75 (2008)

22. E Nekouei, $\mathrm{H}$ Inaltekin, S Dey, Throughput scaling in cognitive multiple access with power and interference constraints. IEEE Trans. Signal Process. 60(2), 927-946 (2011)

23. TStockhammer, M Bystrom, H.264/AVC data partitioning for mobile video communication, in International Conference on Image Processing, vol. 1 (Singapore, October 2004), pp. 545-548

24. MJ Hossain, MS Alouini, VK Bhargava, Hierarchical constellation for multi-resolution data transmission over block fading channels. IEEE Trans. Wireless Commun. 5(4), 849-857 (2006)

25. C Shen, M van der Schaar, Optimal resource allocation for multimedia applications over multiaccess fading channels. IEEE Trans. Wireless Commun. 7(9), 3546 (2008)

26. X Kang, Y-C Liang, A Nallanathan, HK Garg, R Zhang, Optimal power allocation for fading channels in cognitive radio networks: ergodic capacity and outage capacity. IEEE Trans. Wireless Commun. 8(2), 940-950 (2009)

27. X Kang, HK Garg, Y-C Liang, R Zhang, Optimal power allocation for OFDM-based cognitive radio with new primary transmission protection criteria. IEEE Trans. Wireless Commun. 9(6), 2066-2075 (2010)

28. L Zhang, Y Xin, Y-C Liang, HV Poor, Cognitive multiple access channels: optimal power allocation for weighted sum rate maximization. IEEE Trans. Commun. 57(9), 2754-2762 (2009)

29. JB Kim, D Kim, Outage probability achievable diversity order of opportunistic relaying in cognitive secondary radio networks. IEEE Trans. Commun. 60(9), 2456-2466 (2012)

30. R Etkin, A Parekh, D Tse, Spectrum sharing for unlicensed bands. IEEE J. Selected Areas Commun. 25(3), 517-528 (2007)

31. J Lee, H Wang, JG Andrews, Outage probability of cognitive relay networks with interference constraints. IEEE Trans. Wireless Commun. 10(2), 390-395 (2011)

32. $\mathrm{R}$ Zhang, Optimal power control over fading cognitive radio channels by exploiting primary user CSI, in Proceedings of IEEE GLOBECOM 2008 (New Orleans, USA, December 2008), pp. 1-5

33. $Y Y H e, S$ Dey, Power allocation in spectrum sharing cognitive radio networks with quantized channel information. IEEE Trans. Commun. 59(6), 1644-1656 (2011)

34. K Eswaran, M Gastpar, K Ramchandran, Bits through ARQs: spectrum sharing with a primary packet system, in IEEE International Symposium on Information Theory (Nice, France, June 2007), pp. 2171-2175

35. G Ganesan, Y Li, Cooperative Spectrum sensing in cognitive radio networks, (Atlanta, USA, November 2005), pp. 137-143

36. T Cover, J Thomas, Elements of Information Theory, 2nd edn (Wiley, Hoboken, 2006)

37. HA David, HN Nagaraja, Order Statistics, 3rd edn (Wiley, Hoboken, 2003)

38. E Nekouei, H Inaltekin, S Dey, Throughput scaling in cognitive multiple access networks with power and interference constraints, in IEEE International Conference on Communications (Kyoto, Japan, June 2011), pp. 1-6

doi:10.1186/1687-1499-2014-35

Cite this article as: Limmanee et al.: Optimal power policies and throughput scaling analyses in fading cognitive broadcast channels with primary outage probability constraint. EURASIP Journal on Wireless Communications and Networking 2014 2014:35. 